\title{
Characterization of a murine nonalcoholic steatohepatitis model induced by high fat high calorie diet plus fructose and glucose in drinking water
}

\author{
Xue-Jing Liu ${ }^{1}$ Na-Na Duan ${ }^{1,4} \cdot$ Chang Liu $^{1} \cdot$ Chen Niu ${ }^{1} \cdot$ Xiu-Ping Liu ${ }^{2}$ Jian Wu $\oplus^{1,3}$
}

Received: 12 January 2018 / Revised: 20 March 2018 / Accepted: 20 April 2018 / Published online: 29 June 2018

(c) United States \& Canadian Academy of Pathology 2018

\begin{abstract}
There are varieties of murine models of nonalcoholic steatohepatitis (NASH) with different pathophysiologic characteristics. For preclinical assessment, a standardized model would allow comparisons of various pharmacotherapeutic candidates in efficacy, pharmacokinetics, pharmaco-metabolism, and adverse effects under a same system. The present study aims to characterize murine NASH models by comparing end-points of major abnormalities. NASH was induced by feeding high fructose/glucose in drinking water (HF/G), high-fat/calorie diet (HFCD), and in combination (HFCD-HF/G) in mice for 8 or 16 weeks. HF/G feeding caused a minimal fat accumulation and increase in free fatty acids (FFA). In contrast, HFCD-HF/G feeding resulted in a remarkable increase in body weight, subcutaneous and visceral adipose tissue, macrosteatosis with a nearly seven-fold increase in triglyceride and FFA content, accompanied with marked hepatocellular injury, inflammatory responses, fibrosis, and insulin resistance, and represented as typical NASH in histopathology, metabolic, and adipokine profiles in a progressive manner. Meanwhile, mice fed HFCD displayed significant steatosis, necroptosis, fibrosis, insulin resistance, metabolic, and adipokine profiles, and the extent is less than those fed HFCD-HF/G. Significant MCP-1, CCR-2, and NLRP-1/3 activation were found in mice fed HFCD and HFCD-HF/G for 16 weeks, whereas gene expression of CPT-1 and ACOX-1 was down-regulated in these two groups in comparison to the controls. Nuclear receptors, such as SREBP-1c, FXR, LXR- $\alpha$, PPAR- $\alpha$, and PPAR- $\gamma$, were strikingly elevated in the HFCD-HF/G group. In conclusion, feeding HFCD-HF/G resulted in a reliable NASH model in mice with remarkable necroptosis, steatosis, fibrosis, and insulin resistance as well as a disordered profile of lipid metabolism and adipokine, and HFCD caused significant NASH features in histopathology and metabolic profiles only at a late stage. Whereas HF/G feeding barely led to minimal fat accumulation, some changes at molecular levels and metabolic disturbance in mice.
\end{abstract}

\section{Introduction}

The multi-factorial characteristics of nonalcoholic steatohepatitis (NASH) determine variations in pathophysiology and

Electronic supplementary material The online version of this article (https://doi.org/10.1038/s41374-018-0074-z) contains supplementary material, which is available to authorized users.

Jian Wu

jian.wu@fudan.edu.cn

$\triangle$ Xiu-Ping Liu

xpliu1228@fudan.edu.cn

$\triangle$ Chen Niu

chniu@fudan.edu.cn

1 Department of Medical Microbiology, Key Laboratory of Molecular Virology, School of Basic Medical Sciences, Fudan clinical presentation, and no single murine model could mimic all features of NASH patients. In this context, various models of NASH may represent specific manifestations or metabolic disturbances in clinical settings. Therefore, there exists a variety of murine models for NASH, and they are classified into two major categories, i.e. nutritional induction and genetic manipulation [1-3]. For genetic manipulation, majority of the models are identified during studies of specific gene function, and are

University, Shanghai 200032, China

2 Department of Pathology, School of Basic Medical Sciences, Fudan University, Shanghai 200032, China

3 Shanghai Institute of Liver Diseases, Fudan University Shanghai Medical College, Shanghai 200032, China

4 Present address: Stomatological Hospital Affiliated to Soochow University, Suzhou, Jiangsu Province 215005, China 
used to characterize impacts of a specific transgene or knockout. They are certainly fundamental in understanding pathophysiologic function of specific genes and associated pathways, however, may be less relevant to the etiologies and clinical profiles of patients with NASH [4]. Among nutritional dietinduced NASH models, methionine/choline-deficient (MCD) diet and high-fat/calorie diet are most commonly used. Although MCD diet induces steatohepatitis with a relatively shorter duration, loss of body weight, and lack of insulin resistance are the major drawbacks in mimicking manifestations in NASH patients [1,5], especially for those with diabetes, metabolic syndrome; among them more than $70 \%$ are accompanied with NASH [6]. High-fat/cholesterol diet causes an increase in body weight, obesity, hepatic steatosis with minimal inflammation, fibrosis, and insulin resistance within a period of 2 months, and do not exhibit marked hepatocellular death, inflammation, and progression to hepatic fibrosis [7]. High fructose alone may lead to some extent of fat accumulation and inflammatory response; however, it hardly causes obvious steatohepatitis [8], and is often supplemented in addition to high-fat diet [3]. There are other diets, such as transfat (conjugated linoleic acid, CLA), which resulted in marked steatohepatitis and insulin resistance [9], or cholinedeficient amino acid-defined diet that also induces steatohepatitis without loss in body weight in mice [10]. Moreover, to better mimic less physical activities, high-fat diet plus sedentary lifestyle caused obesity syndrome with typical NASH presentations within 6 months [11].

High-fat/calorie diet is the most common dietary method to induce NASH in mice, and the model mimics Western fast food dietary composition. However, since there are many different recipes, feeding combination, and duration, it is in high demand to standardize a protocol to establish a reliable model for better comparison in terms of assessment of pharmaceutical candidates or pathophysiologic investigation between different models. Thus, we compared high-fat/calorie diet alone, high fructose/glucose in drinking water, and their combination in extent of steatosis, hepatocellular injury, inflammatory responses, insulin resistance, fibrotic progression, and metabolic disturbance with 16 weeks of feeding. Our data suggest that high-fat/calorie diet plus high fructose/glucose in drinking water is optimal for the development of significant steatosis, necroptosis, inflammatory reaction, insulin resistance, and fibrotic progression, and represents a valuable model for pharmacologic intervention or pathophysiologic investigation.

\section{Materials and methods}

\section{Animals and diets}

Male C57BL6J mice aged 6-8 weeks from Nanjing Biomedical Research Institute of Nanjing University (Nanjing,
China) were housed in a specific pathogen-free facility with 12-h light/dark cycle at $22^{\circ} \mathrm{C}$ in Fudan University Experimental Animal Center (Shanghai, China). After a 2-week acclimatization, mice were randomly divided into four groups ( $n=8$ per group): (1) standard pellet diet and tap water (Control); (2) pellet diet plus high fructose/glucose (23.1 g L-fructose plus $18.9 \mathrm{~g}$ L-glucose in 11 of tap water, cat. no. F3510, G8270; Sigma-Aldrich, St Louis, MO, USA) in drinking water (HF/G) [12]; (3) high-fat/calorie diet (cat. no. D12492; Research Diet, New Brunswick, NJ, USA) with tap water (HFCD) (for nutritional composites, please see Supplemental Table 1); (4) high-fat/calorie diet plus high fructose/glucose in drinking water (HFCD-HF/G) as we reported recently [13]. The body weight and diet consumption of mice was monitored weekly. Animal experimental procedures were approved by the Animal Ethic Committee of Fudan University School of Basic Medical Sciences, and performed following the NIH Guidelines of Experimental Animal Handling and Use. Mice were sacrificed after 8 or 16 weeks of feeding, the liver and serum were collected, and stored at $-80^{\circ} \mathrm{C}$ for histopathologic, biochemical, and molecular analyses.

\section{Quantitative computed tomography scan of whole body}

To visualize whole-body adipose tissue accumulation and distribution, including subcutaneous, visceral, and brown fat, a whole-body micro-computed tomography (CT) scan was performed at 16 weeks of feeding. Detailed threedimensional images of mouse internal structure were obtained by high-resolution X-ray microCT scanning (Quantum FX; PerkinElmer, Hopkinton, MA, USA) [14]. Mice were anesthetized with $2.5-3 \%$ isoflurane and positioned on the scan platform. X-ray source was set to a current of $100 \mu \mathrm{A}$ and a voltage of $80 \mathrm{kVp}$. The scan was initiated from a nearby computer terminal and totally lasted for 4 min per mouse. CT images were taken with a field of view of $70 \mathrm{~mm} \times 40 \mathrm{~mm}$ and a voxel size of $144 \mu \mathrm{m}$. Image segmentation was conducted using a volume-editing tool, and volumes were quantified using the region of interest module within the software package (AnalyzeDirect, Overland Park, KS, USA). The adipose content and distribution were calculated with a software (Analyze 12.0) as we reported previously [13].

\section{Intraperitoneal glucose tolerance test}

To assess insulin resistance status, intraperitoneal glucose tolerance test (IGTT) was performed after 8 and 16 weeks of feeding. Mice were injected intraperitoneally with glucose $(2.5 \mathrm{~g} / \mathrm{kg}$ body weight $)$ after being fasted for $15 \mathrm{~h}$. Glucose level was measured at $0,30,60,90$, and $120 \mathrm{~min}$ 
after injection by a glucometer (cat. no. HGM-112; Omron Company, Dalian, China) as we reported previously [13].

\section{Serological analysis}

Mouse blood was collected from eye orbit bleeding, and serum was separated by centrifugation (2000 $g$ for $10 \mathrm{~min}$ at $4{ }^{\circ} \mathrm{C}$ ). Serum levels of triglyceride (TG), total cholesterol (TC), low-density lipoprotein (LDL), high-density lipoprotein (HDL), total bilirubin (TBIL), total bile acid (TBA), alanine transaminase (ALT), and aspartate transaminase (AST) were determined by an automated biochemical analyzer (cat. no. AU680; Beckman Coulter Company, Fullerton, CA, USA).

Serum insulin level was measured with a mouse insulin ELISA kit (cat. no. EZRMI-13K; Millipore, Billerica, MA, USA). Index of homeostasis model assessment insulin resistance (HOMA-IR) was calculated following a formula: HOMA-IR $=($ fasting serum insulin $(\mathrm{mU} / \mathrm{l}) \times$ fasting serum glucose $(\mathrm{mmol} / \mathrm{l})) / 22.5$ as we reported previously [13].

\section{Liver biochemical analysis}

Liver TG and TC levels in mouse liver were measured with commercially available detection kits (cat. no. E1013 and E1015; Applygen Technologies, Beijing, China) after being homogenized by a homogenizer (cat. no. SKSI; Shanghai Biheng Biotechnology Company, Shanghai, China). Liver levels of free fatty acids (FFA) were measured by a colorimetric method using commercially available kit (cat. no. A042-1; Nanjing Jiancheng Bioengineering institute, Nanjing, China). Hepatic hydroxyproline content was measured by the acid hydralyzation method following a protocol provided by the manufacturer (cat. no. A03; Nanjing Jiancheng Bioengineering Institute) [15].

\section{Liver histopathologic examination}

Fresh mouse liver was fixed in $10 \%$ neutral formalin, embedded in paraffin, and sectioned in $4 \mu \mathrm{m}$ thickness. Liver sections were stained in hematoxylin and eosin (H\&E), and were examined to semi-quantitatively assess the NAFLD activity score (NAS) based on histological features by a board-certified pathologist blind to the experimental protocol. The score is in three categories and classified into: steatosis (0-3), lobular inflammation (0-2), and hepatocellular ballooning (0-2). If NAS of a liver specimen is $\geq 5$, the section is defined as "NASH". If NAS is smaller than 3, it represents as "non-NASH" [16]. The extent of liver fibrosis was assessed after Masson's Trichrome staining (cat. no. HT-15; Sigma-Aldrich), and semi-quantitatively scored between 0 and 4 following the criteria as reported previously $[13,16]$. Hepatocellular apoptosis in the liver was determined after TdT-mediated dUTP nick-end labeling (TUNEL) staining (cat. no. 11684817910; Roche, Indianapolis, IN, USA), and the TUNEL-positive nuclei were counted under a high-power field (HPF, $\times 40$ objective field) in 10 randomly chosen fields [17]. Frozen liver sections in $7-\mu \mathrm{m}$ thickness were fixed in $4 \%$ neutralized paraformaldehyde in phosphate-buffered saline (PBS) and stained with Oil Red-O (ORO) (cat. no. O0625, SigmaAldrich) to visualize fat droplet deposition in hepatocytes. All stained sections were photographed under a $\times 200$ field of light microscopy (ECLIPSE Ci-L; Nikon Corporation, Tokyo, Japan) and semi-quantification by Imaged J (NIH, USA).

\section{Immunofluorescent staining}

Frozen liver sections were fixed in $4 \%$ neutralized paraformaldehyde in PBS for $15 \mathrm{~min}$ and then washed in $1 \times$ PBS for $5 \mathrm{~min}$ for three times. Sections were treated with $3 \%$ Triton X-100 (cat. no. V900502, VETEC; Sigma-Aldrich) to increase the permeability of the primary antibody into cytosol, blocked with $3 \%$ bovine serum albumin (BSA), incubated with primary antibody (1:50) (Supplemental Table 3) diluted with $1 \% \mathrm{BSA}$ at $4{ }^{\circ} \mathrm{C}$ overnight, and washed with PBS as described above. Thereafter, they were incubated with fluorescence (Flexa 488 or 594)-conjugated secondary antibody $(1: 200)$ for $2 \mathrm{~h}$ at $37^{\circ} \mathrm{C}$, and washed for three times. Nuclei were counterstained with 4'6-diamidino2-phenylindole (cat. no. C1005; Beyotime Biotechnology, Haimen, China) [18]. All sections were sealed with glycerin, examined, and photographed in HPF $\times 40$ objective field under a confocal microscope (cat. no. TCS SP8, Leica, Germany).

\section{Quantitative real-time PCR}

Total RNA was isolated from mouse liver tissue using TRIZOL reagent, followed a standard protocol, and reversely transcribed to cDNA using a Primer-Script RT kit (cat. no. RR037A; Takara Bio, Dalian, China). Quantitative reverse transcriptase polymerase chain reaction (RT-PCR) was carried out with Power SYBY green PCR Master Mix (cat. no. 4368708, Applied Biosystems, USA), using an Eppendorf realplex cycle (cat. no. realplex4, Eppendorf, Germany). Relative target gene level was analyzed using $2^{-\Delta \Delta \mathrm{Ct}}$ method and normalized by house-keeping $\beta$-actin gene control [17]. Primers used in PCR are listed in Supplementary Table 2.

\section{Western blot analysis}

Liver protein was extracted with RIPA Lysis Buffer (cat. no. P0013B; Beyotime Biotechnology) and quantitated 
Fig. 1 Distribution of adipose tissue and body weight changes. A-C Body and liver weight in different groups at $8(n=3)$ and 16 weeks $(n=5)$. D MicroCT 3D images of subcutaneous, visceral, and brown adipose tissue distribution at 16 weeks $(n=3)$. E Quantification of different adipose tissue content with a software $(n=3)$. $* p<0.05$ and $* * p<0.01$ compared to the controls. a $p<0.05$ and aap $<0.01$ compared to HF/G. $\mathrm{b} p<0.05$ and $\mathrm{bb} p<0.01$ compared to HFCD. Subcu subcutaneous, Viscera visceral
A

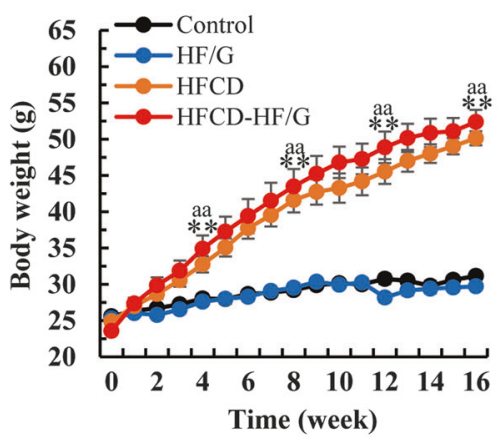

$\mathbf{B}$

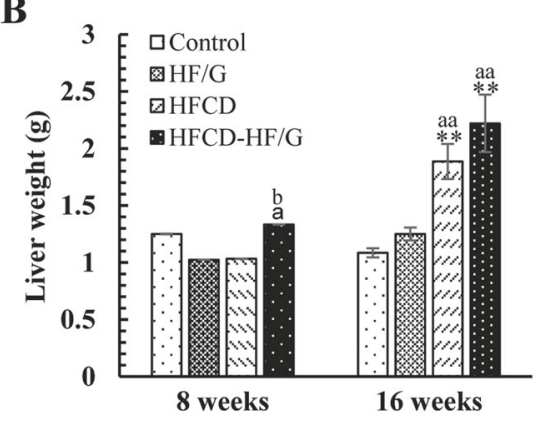

C

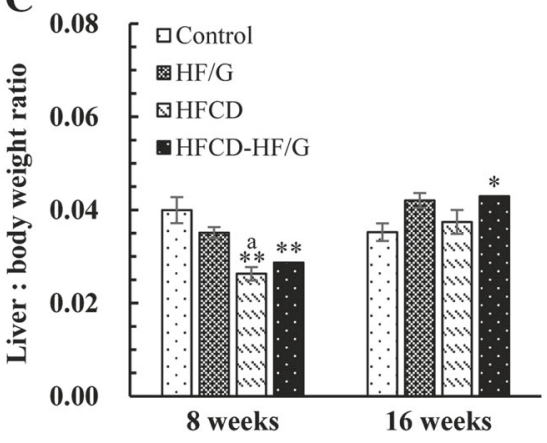

D Subcutaneous fat Viscera fat

Brown fat
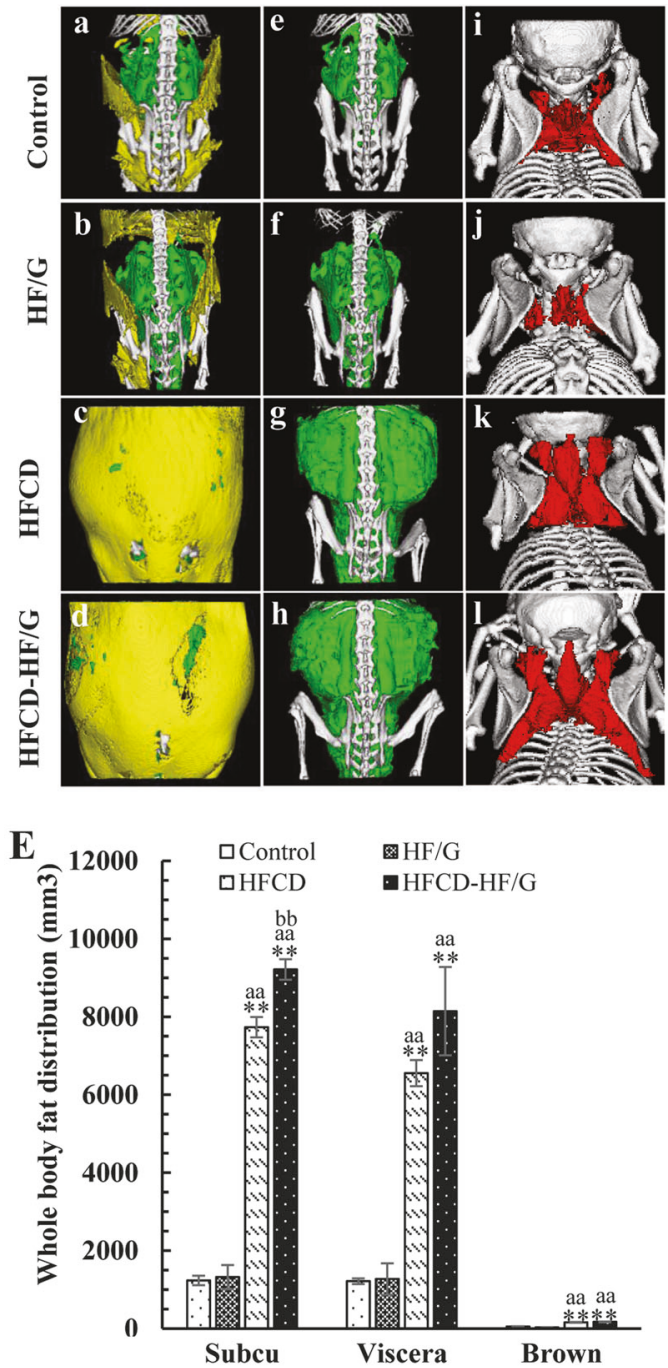

using a BCA assay kit (cat. no. 23227; Thermo Fisher Scientific, Waltham, MA, USA). Denatured protein was separated by SDS-PAGE electrophoresis and transferred onto polyvinylidene fluoride (PVDF) membrane (cat. no. IPVH00010; Millipore). The membranes were blocked with 5\% non-fat milk (cat. no. NB0669; Sangon Biotech, Shanghai, China) in Tris-HCl-buffered saline solution plus Tween 20 (TBST) for $2 \mathrm{~h}$ at room temperature, incubated overnight at $4{ }^{\circ} \mathrm{C}$ with primary antibodies, and followed by horseradish peroxidase-conjugated secondary antibodies at room temperature for $1 \mathrm{~h}$. Target protein bands were visualized using the ECL kit (cat. no. WBKLS0500; Millipore) and chemiluminescent imaging system (cat. no. 14T12NPFL16-348; Shanghai Tanon Technology Company, Shanghai, China), and the densitometry was analyzed by Image J software [19]. Antibodies used in Western blot analysis are listed in Supplementary Table 3.

\section{Statistical analysis}

All data were expressed as mean \pm SEM. Statistical analysis was performed with SPSS 17.0 software. After normal distribution test, the ANOVA variance test was used to compare between groups, and multiple comparisons between two given groups were completed by the least significant difference (LSD) test. A value of $p<0.05$ was considered to be statistically significant.

\section{Results}

\section{Fat accumulation in mice fed different diets}

As shown in Fig. 1, mice in HFCD and HFCD-HF/G groups exhibited an accelerated gain in body weight (Fig. 1A) and liver weight (Fig. 1B) over time; those in HF/ 
A
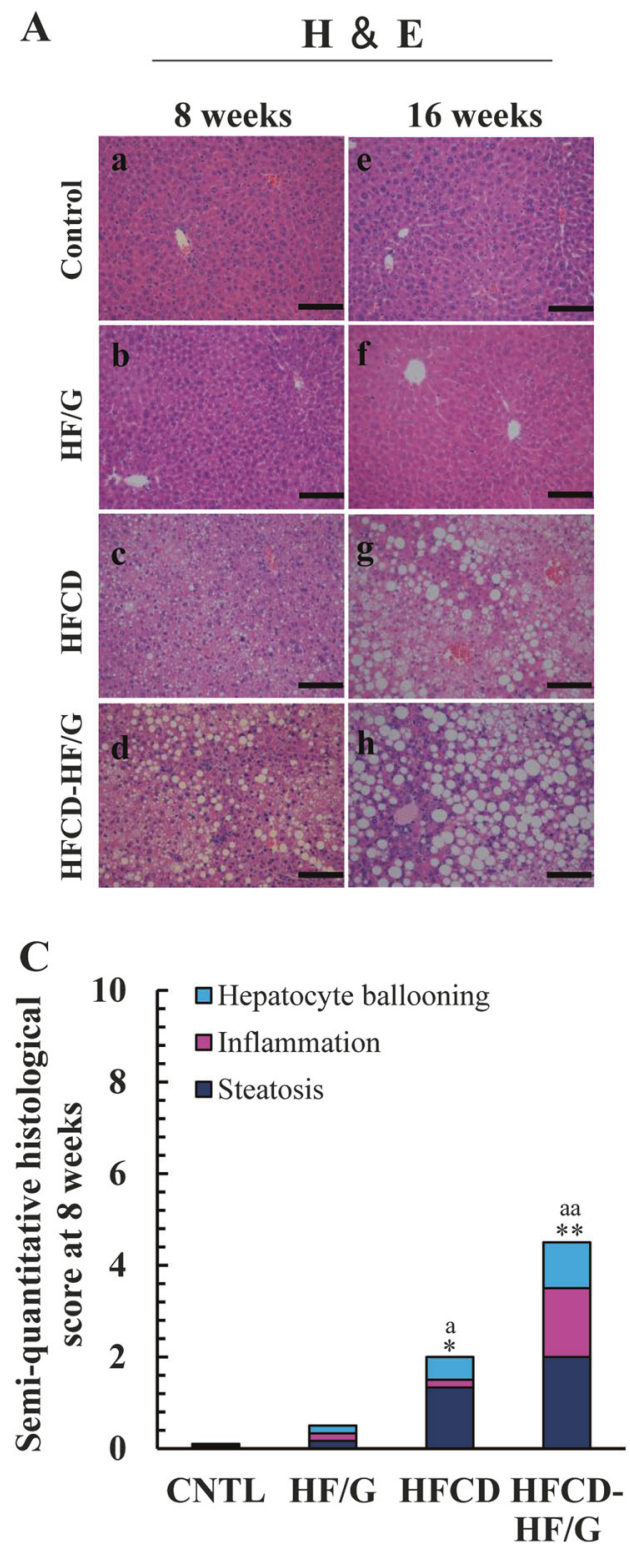

Fig. 2 Liver histopathology of mice fed different diet. A Representative micrographs of liver histology in H\&E staining at $8(\mathrm{a}-\mathrm{d})(n=3)$ and 16 weeks $(\mathrm{e}-\mathrm{h})(n=5)$. Images were taken at original magnification $(\times 200)$. Scale bars $=100 \mu \mathrm{m}$. B Representative micrographs of Oil Red-O staining at $8(\mathrm{a}-\mathrm{d})$ and 16 weeks $(\mathrm{e}-\mathrm{h})(n=3)$. Images were

$\mathrm{G}$ and control groups remained in a normal increase trend in body weight. The ratio of liver weight/body weight (Fig. 1C) declined at 8 weeks due to an accelerated body weight increase in HFCD and HFCD-HF/G groups with a similar liver gain trend among these four groups. The ratio increased significantly in the HFCD-HF/G group than the control group at 16 weeks as a result of an accelerated increase in the liver weight in this group (Fig. 1B). MicroCT scanning quantification demonstrated that the volume of subcutaneous (6.2-, 7.5-fold), visceral (5.4-, 6.7fold), and brown (3.0-, 3.3-fold) adipose tissue in HFCD
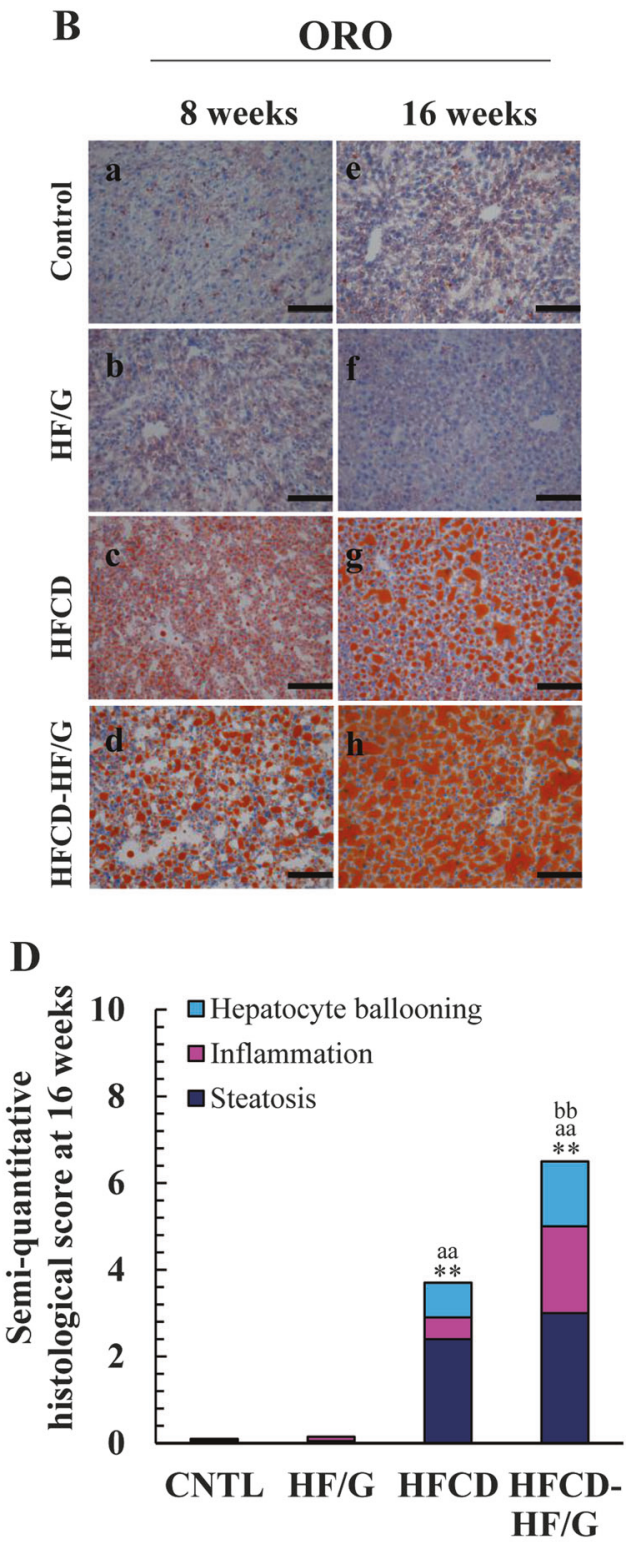

taken at original magnification $(\times 200)$. Scale bars $=100 \mu \mathrm{m}$. C, D Semi-quantitative score of histopathology at $8(\mathbf{C})(n=3)$ and 16 (D) weeks $(n=5)$. $* p<0.05$ and $* * p<0.01$ compared to the controls. a $p<0.05$ and aap $<0.01$ compared to HF/G. b $p<0.05$ and bb $p<0.01$ compared to HFCD. CNTL Control

and HFCD-HF/G groups at 16 weeks were remarkably higher than the control and HF/G groups (Fig. 1D, E). Obviously, the subcutaneous and visceral adipose tissue increased much more than brown adipose tissue in the HFCD and HFCD-HF/G groups. Moreover, the volume of subcutaneous adipose tissue in the HFCD-HF/G group was higher than that in the HFCD group (Fig. 1E). In summary, high-fat feeding resulted in significant body fat accumulation and gain in liver weight, and the supplementation of high fructose/glucose in drinking water accelerated the development of obesity. 
Fig. 3 Variables of liver injury in mice fed different diet. A Representative micrographs of TUNEL staining at $8(\mathrm{a}-\mathrm{d})$ and 16 weeks $(\mathrm{e}-\mathrm{h})(n=3)$. Images were taken at original magnification $(\times 200)$. Scale bars $=100 \mu \mathrm{m}$. B Apoptotic cell count in liver sections from three mice in each group $(n=3)$. $\mathbf{C}, \mathbf{D}$ Serum levels of ALT $(\mathbf{C})$ and AST $(D)$ at $8(n=3)$ and 16 weeks $(n=5) . * p<0.05$ and $* * p<0.01$ compared to the controls. ap $<0.05$ and aap $<$ 0.01 compared to HF/G. $\mathrm{b} p<$ 0.05 and $\mathrm{bb} p<0.01$ compared to HFCD. $A L T$ alanine aminotransferase, $A S T$ aspartate aminotransferase, $T N F-\alpha$ tumor necrosis factor $\alpha, M C P-1$ monocyte chemoattractant protein-1, CCR-2 C-C chemokine receptors-2, Adpnt adiponectin
A

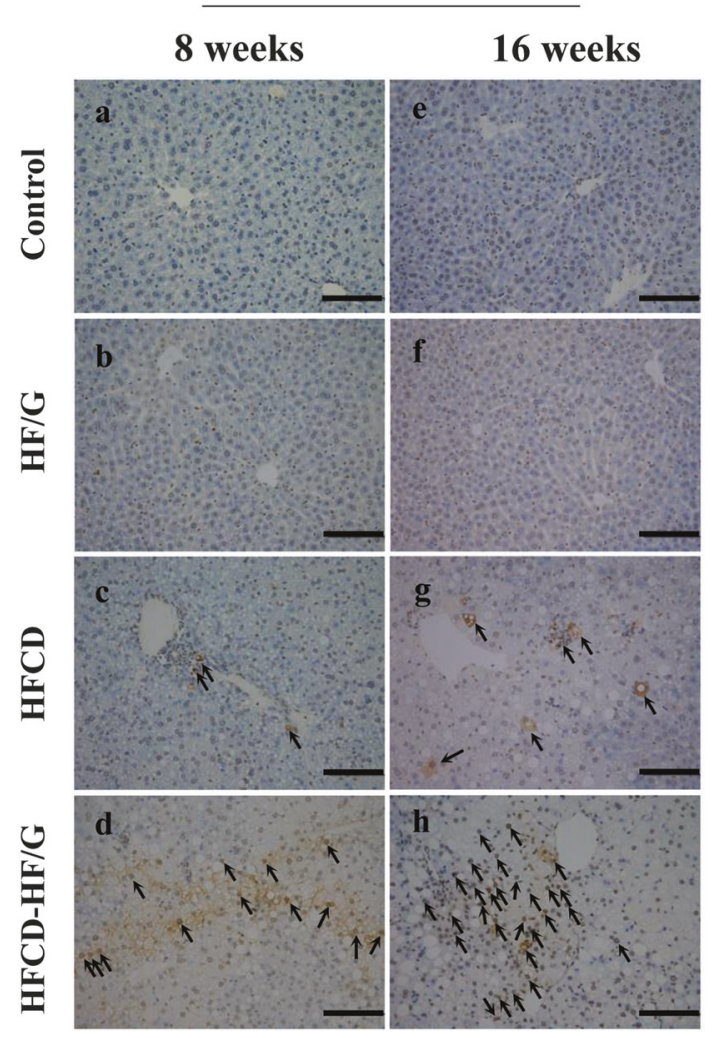

TUNEL

$\mathbf{E}$

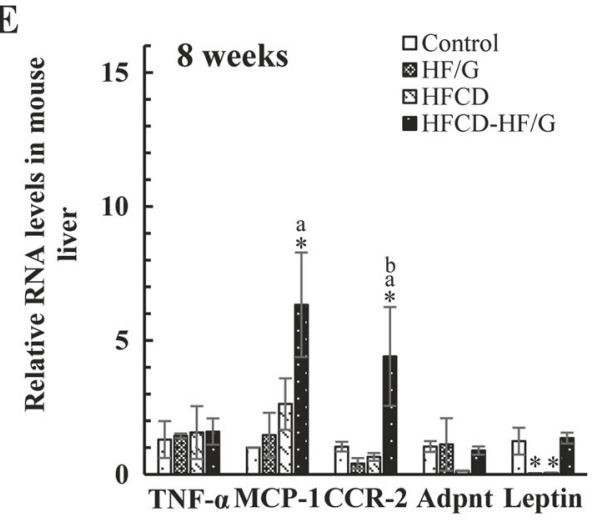

B
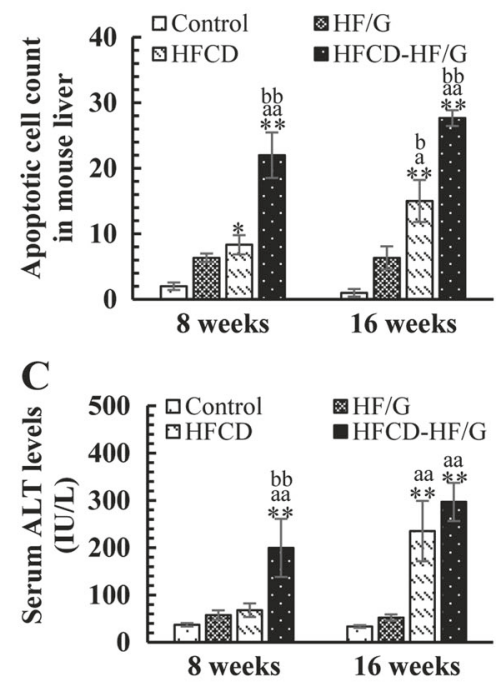

D

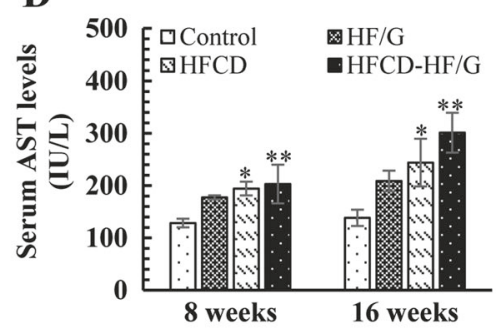

\section{Hepatocellular steatosis in mice fed different diets}

Hepatocytes in the HFCD and HFCD-HF/G groups appeared to be steatotic in various degrees at 8 and 16 weeks as shown by H\&E and ORO staining (Fig. 2A, B), whereas steatosis as shown in micro, macro, or mixed size of lipid droplets in the HFCD group was less severe in comparison to the HFCD-HF/G group. Only micro-lipid droplets were observed in the liver of mice fed with $\mathrm{HF} / \mathrm{G}$ in drinking water. The semi-quantitative score of steatosis and semi-quantitative analysis of Oil Red-O-positive staining area further confirmed the steatotic findings at 8 and 16 weeks (Fig. 2C, D, Supplemental Fig. 1C) and is consistent with TG quantitation (Fig. 4B). Liver TG content in the HFCD and HFCD-HF/G groups was significantly increased compared to that in the control group at 8 (3.4-, 4.0-fold) and 16 weeks (4.3-, 6.6-fold) (Fig. 4A, B), and was increased slightly in the $\mathrm{HF} / \mathrm{G}$ group at 8 weeks (Fig. 4A), and no further increase was seen at 16 weeks. Liver levels of total FFA in different groups displayed an increasing tend similar to liver TG levels (Fig. 4B). In summary, the most severe steatosis was induced by the HFCD-HF/G diet, and severe steatosis by HFCD group, and the liver TG and FFA content further confirmed the steatotic changes. On contrast, there was mild steatosis in the liver of mice fed HF/G in drinking water. 
Fig. 4 Variables of liver biochemical and lipid panel. A, B Liver total cholesterol (TC), triglyceride (TG), and FFA content at $8(\mathbf{A})$ and 16 weeks (B). C, D Serum levels of TG, TC, LDL, and HDL at 8 (C) and 16 weeks (D). E, F Serum levels of TBIL (E) and TBA $(\mathbf{F})$ at 8 and 16 weeks. A-F $n=3$ for 8 weeks, $n=5$ for 16 weeks. $* p<0.05$ and $* * p$ $<0.01$ compared to the controls. a $p<0.05$ and aap $<0.01$ compared to HF/G. $\mathrm{b} p<0.05$ and $\mathrm{bb} p<0.01$ compared to HFCD. $T C$ total cholesterol, $T G$ triglyceride, FFA free fatty acid, $L D L$ low-density lipoprotein, $H D L$ high-density lipoprotein, TBIL total bilirubin, TBA total bile acid
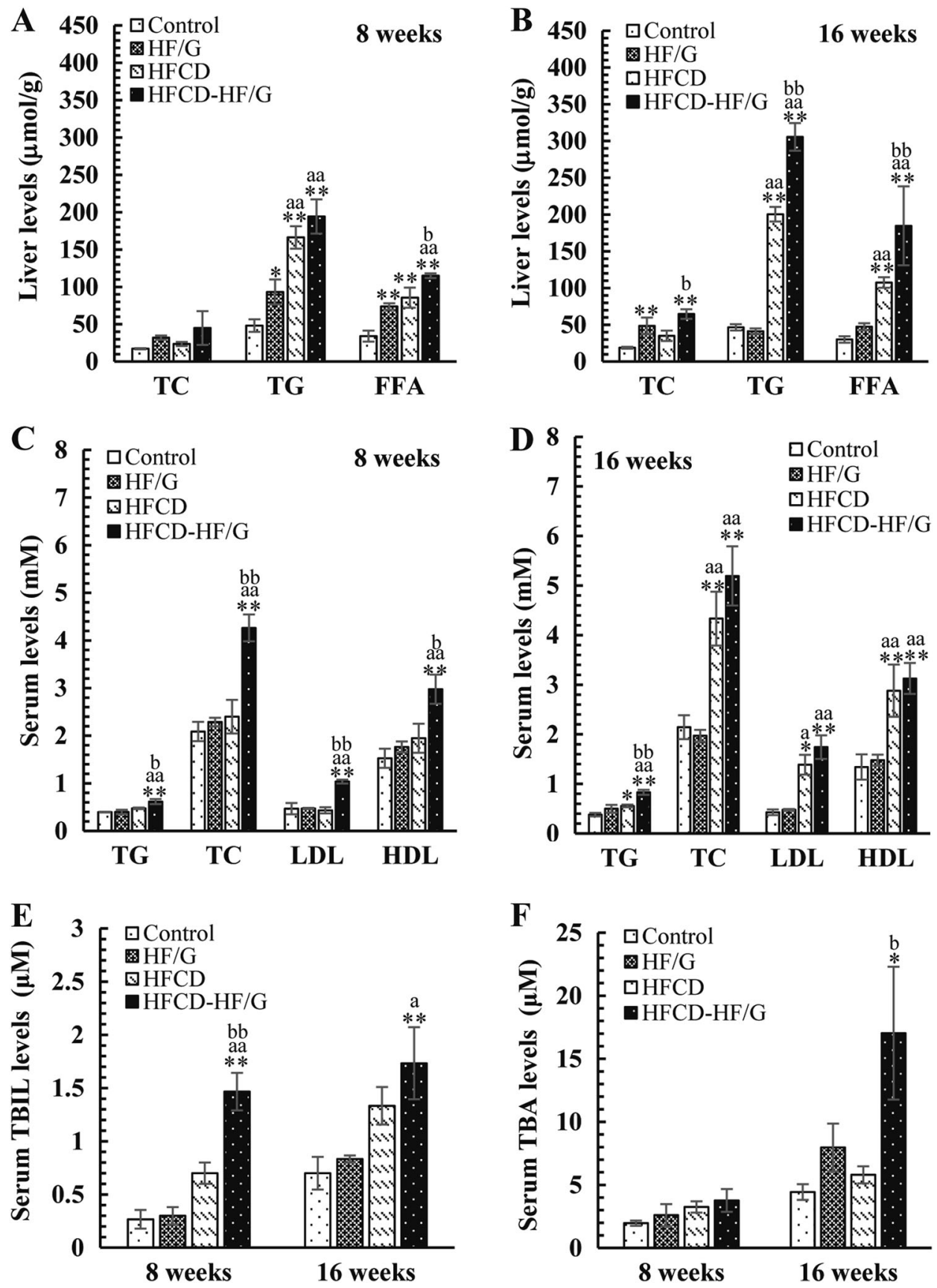

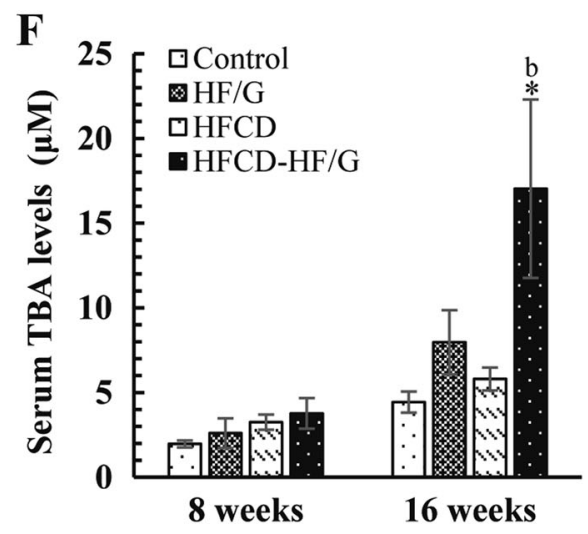

\section{Liver injury and inflammation in mice fed different diets}

H\&E (Fig. 2A) staining revealed that there was severe hepatocellular ballooning and injury in the HFCD and HFCD-HF/G groups, whereas there hardly was a difference in hepatocellular ballooning and injury between $\mathrm{HF} / \mathrm{G}$ and control groups. Infiltration of inflammatory cells was found to be in the portal triads in both HFCD and HFCD-HF/G groups. Semi-quantitative scores of hepatocellular ballooning and inflammation further confirmed this finding
(Fig. 2C, D). Hepatocellular apoptosis was determined by TUNEL staining (Fig. 3A). A minimal apoptotic cell count was found in the HF/G group $(p>0.05)$. More apoptotic cells were found in the HFCD-HF/G group than the control and HF/G group at both 8 (d vs. a) and 16 weeks (h vs. e), and there was an elevated apoptotic cell count in the HFCD group in comparison to the control at 16 weeks (Fig. 3B). Serum ALT levels in the HFCD-HF/G group were extremely (5.4-, 8.8-fold) higher than the control group at 8 and 16 weeks, and a 7-fold ALT increase in the HFCD group at 16 weeks was noted, whereas it was barely elevated in the 
Fig. 5 Insulin resistance status in different groups. A, B

Intraperitoneal glucose tolerance test (IGTT) at $8(\mathbf{A})$ and $16(\mathbf{B})$ weeks. C, D Serum insulin levels and index of homeostasis model assessment of insulin resistance (HOMA-IR) at $8(\mathbf{C})$ and 16 (D) weeks. E, F qRTPCR analysis of INSR, IRS-1, and IRS-2 at $8(\mathbf{E})$ and $16(\mathbf{F})$ weeks. A-F $n=3$ for 8 weeks and $n=5$ for 16 weeks. G Relative pIRS-1 and pAkt protein levels were determined by western blot analysis at 8 and 16 weeks $(n=3)$. ${ }^{*} p<0.05$ and $* * p<0.01$ compared to the controls. a $p<0.05$ and aap $<$ 0.01 compared to HF/G. $\mathrm{b} p<$ 0.05 and $\mathrm{bb} p<0.01$ compared to HFCD. INSR insulin receptor, IRS-1 insulin receptor substrate1, IRS-2 insulin receptor substrate-2, Akt PKB (protein kinase B). pIRS1 phosphorylation of IRS-1 at serine 307, pAkt phosphorylation of Akt-1 at serine 473
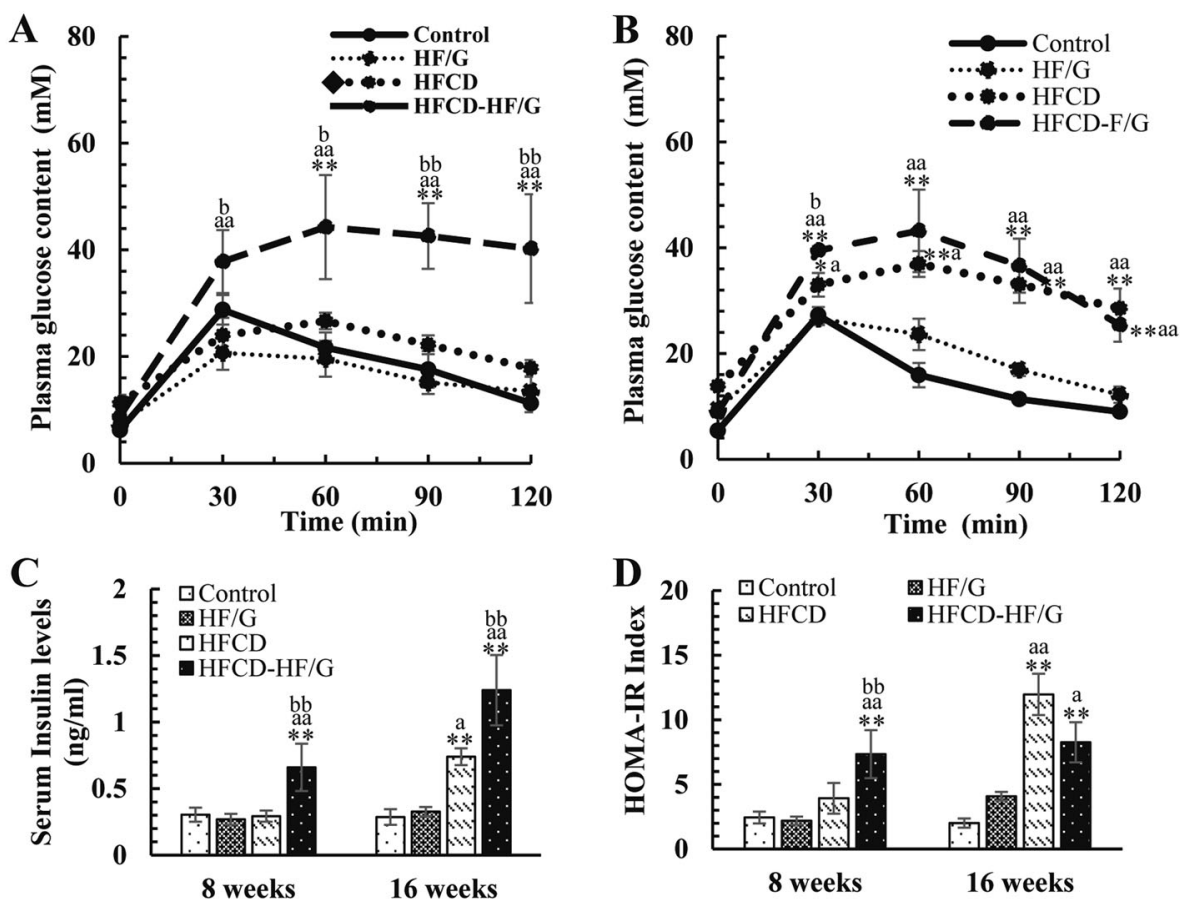

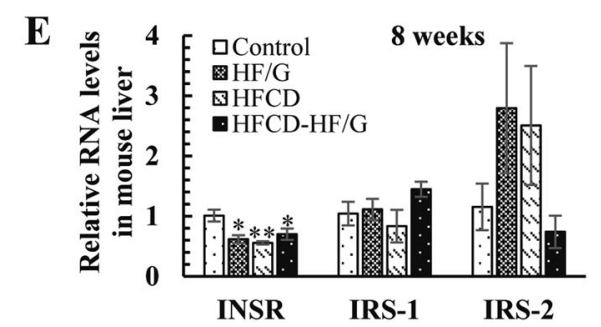

\section{G}

8 weeks

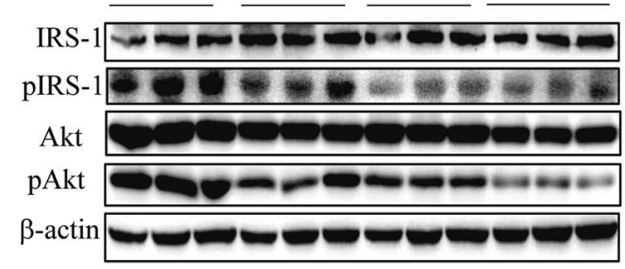

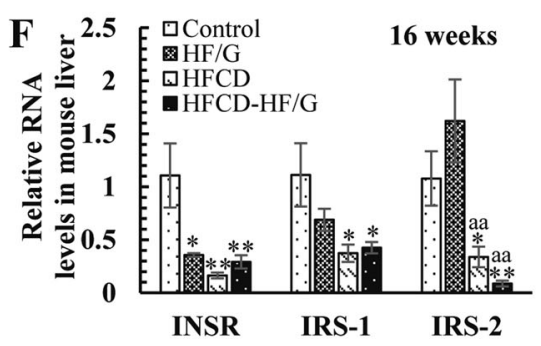

16 weeks

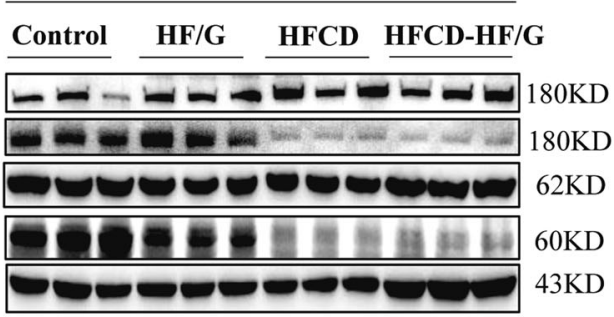

HF/G group compared to the control group (Fig. 3C). Serum AST levels in the HFCD and HFCD-HF/G groups were obviously higher than the control group at 8 and 16 weeks (1.8-, 2.2-fold), and were mildly increased in the HF/G group $(p>0.05)$ (Fig. 3D). Along with an ALT and AST increase, serum levels of TBIL in the HFCD and HFCD-HF/G groups were markedly increased compared to the control group at 8 and 16 weeks (Fig. 4E). In summary, profound liver necroptosis was documented in both HFCD and HFCD-HF/G groups, especially in the HFCD-HF/G group, and no significant hepatocellular injury was noted in mice fed $\mathrm{HF} / \mathrm{G}$ in drinking water.

\section{Changes of inflammatory gene, cytokine, or adipokine profile}

To investigate the inflammatory response, mRNA levels of monocyte chemoattractant protein-1 (MCP-1) and C-C chemokine receptor type-2 (CCR-2) were determined by quantitative RT-PCR. It was found that they were both increased remarkably in the HFCD-HF/G group compared to the control group at 8 and 16 weeks (Fig. 3E, F), but not elevated significantly in the HF/G and HFCD groups. The mRNA levels of tumor necrosis factor- $\alpha$ (TNF- $\alpha$ ) were increased only in the HFCD group at 
16 weeks, but were not increased significantly in other groups (Fig. 3F).

To investigate the changes of adipokine profile, mRNA levels of adiponectin and leptin were determined in mouse liver, and it is evident that both leptin and adiponectin in the HFCD-HF/G group did not change significantly at 8 weeks, whereas were markedly down-regulated compared to the control group at 16 weeks (Fig. 3E, F). The mRNA levels of adiponectin and leptin in the HFCD group were lower than the control group at both time points (Fig. 3E, F). The mRNA levels of leptin in the HF/G group decreased at both time points; however, the adiponectin mRNA levels went down only at 16 weeks (Fig. 3E, F) in comparison to the control group $(p<0.05)$. In summary, feeding HFCD-HF/G diet dramatically increased the gene expression levels of MCP-1 and CCR-2 throughout the whole duration. In contrast, gene expression levels of adiponectin and leptin were down-regulated in these mice at 16 weeks. HFCD feeding exerted moderate effects on down-regulating these two adipokine genes, and raised $\mathrm{TNF}-\alpha$ gene level at 16 weeks $(p<0.05)$.

\section{Disordered lipid metabolism in mice under different diets}

Liver TC (including free cholesterol and cholesterol ester) levels were obviously higher than the controls in $\mathrm{HF} / \mathrm{G}$, HFCD, and HFCD-HF/G groups at 8 and 16 weeks; serum cholesterol levels were elevated significantly in the HFCD$\mathrm{HF} / \mathrm{G}$ and HFCD groups only at 16 weeks (Fig. 4A-D). Serum TG levels exhibited an apparent elevation in mice fed HFCD-HF/G (Fig. 4C, D). Serum levels of LDL and HDL were significantly increased in the HFCD-HF/G group at 8 and 16 weeks compared to the controls (Fig. 4C, D), whereas they were increased only at 16 weeks in the HFCD group (Fig. 4D). Serum levels of TBA in the HFCD-HF/G group were much higher than the control group at 16 weeks (Fig. 4F). There was no obvious difference in serum levels of LDL and HDL between HF/G and the control group (Fig. 4C, D), whereas serum TBA levels were elevated mildly in the HF/G group at 16 weeks $(p>0.05)$ (Fig. 4F). In summary, along with marked lipid accumulation in the liver, serum levels of lipid panel of TG, TC, LDL, and HDL, as well as TBA were elevated in the HFCD-HF/G group at both 8 and 16 weeks of feeding, and a late increase in these variables was seen in the HFCD group. Only a liver TC increase was noted in mice fed HF/G in drinking water.

\section{Insulin resistance in mice fed different diets}

To assess glucose tolerant status, IGTT was performed 2 days before sacrifice at 8 and 16 weeks of feeding, plasma glucose levels in HFCD-HF/G groups were markedly elevated and sustained at high levels at 8 and 16 weeks (Fig. 5A, B). Plasma glucose levels were significantly elevated and sustained only at 16 weeks in the HFCD group (Fig. 5B). In contrast, plasma glucose levels in the HF/G and control groups increased $30 \mathrm{~min}$ after glucose injection and returned to normal levels quickly (Fig. 5A, B). As shown in Fig. 5C, D, serum insulin levels and HOMA-IR index of the HFCD-HF/G group were elevated notably compared to the controls, and they were increased only at 16 weeks in the HFCD group. However, there was no difference in fast insulin levels and MOMA-IR index between the $\mathrm{HF} / \mathrm{G}$ and control groups. Relative RNA levels of insulin receptor (INSR) in HF/G, HFCD, and HFCD-HF/G groups were markedly decreased compared to the controls at 8 and 16 weeks (Fig. 5E, F). Relative RNA levels of insulin receptor substrate-1 (IRS-1) and IRS-2 in HFCD and HFCD-HF/G groups were significantly reduced compared to the control group at 16 weeks (Fig. 5F) although IRS-1 was not notably changed in total protein extract (Fig. 5G). In consistent with insulin signaling defect, phosphorylation of IRS-1 at serine 307 residue was remarkably reduced in mice fed HFCD and HFCD-HF/G diet at both 8 and 16 weeks, and much more suppression in those fed HFCD$\mathrm{HF} / \mathrm{G}$ diet compared to the controls. However, there was no significant changes in IRS-1phosphorylation status in mice fed $\mathrm{HF} / \mathrm{G}$ alone, consistent with its RNA and protein levels (Fig. 5G). The protein phosphorylation of insulin receptor signaling molecule, Akt at the serine 473 residue, was strikingly minimized in both HFCD and HFCD-HF/G groups compared to the control at 8 and 16 weeks (Fig. 5G). Densitometric analysis of Western blotting imaging of pIRS-1 over IRS-1 and pAkt over Akt is shown in Supplemental Fig. 1A, B and further confirms the findings. In summary, no significant insulin resistance occurred in mice fed $\mathrm{HF} / \mathrm{G}$ alone; insulin resistance was worsened in an increasing order of HFCD and HFCD-HF/G groups; and profound insulin resistance was seen in HFCD at 16 weeks, and HFCD-HF/G at 8 and 16 weeks with progressive deterioration. Impaired insulin receptor signaling as demonstrated by suppressed phosphorylation of IRS-1 and Akt seems to be responsible for the deteriorating insulin sensitivity in mice fed HFCD over 16 weeks and HFCD$\mathrm{HF} / \mathrm{G}$ diets in both 8 and 16 weeks.

\section{Hepatic fibrogenesis in mice in different diets}

To determine the extent of fibrosis, Masson's Trichrome staining of mouse livers was performed. It is evident that collagenous fibrils were deposited in the portal triads and pericellular space in the HFCD-HF/G group at 8 and 16 weeks (Fig. 6A), which was verified by semiquantitative fibrosis score, quantitation of liver hydroxyproline content, and semi-quantitative analysis of 
Fig. 6 Liver fibrosis and hepatic collagen deposition. A

Representative micrographs of Masson Trichrome staining at 8 (a-d) and 16 weeks (e-h). Images were taken at original magnification $(\times 200)$. Scale bars $=100 \mu \mathrm{m}$. B Semiquantitative score of hepatic fibrosis at 8 and 16 weeks. C Liver hydroxyproline content $(\mu \mathrm{g} / \mathrm{mg})$ at 8 and 16 weeks. A-C $n=3$ for 8 weeks and $n=5$ for 16 weeks. D Western blot analysis of $\alpha$-SMA protein levels at 8 and 16 weeks $(n=3)$. $\mathbf{E}, \mathbf{F}$ mRNA levels of procollagen type-I (Col-I $\alpha 1$, Col-I $\alpha 2)$, procollagen type-III (Col-III 1 1), and tissue inhibitor of metalloproteinase-1 (TIMP-1) in mice under different diet feeding for $8(\mathbf{E})$ and $16(\mathbf{F})$ weeks $(n=3) . * p<0.05$ and $* * p<0.01$ compared to the controls. a $p<0.05$ and aap $<$ 0.01 compared to HF/G. $\mathrm{b} p<$ 0.05 and $\mathrm{bb} p<0.01$ compared to HFCD. $\alpha$-SMA smooth muscle $\alpha$-actin, Col-I $\alpha 1$ procollagen type-I $\alpha 1$, Col-I $\alpha 2$ procollagen type-I $\alpha 2$, Col-III $\alpha 1$ procollagen type-III $\alpha 1, T I M P-1$ tissue inhibitor of metalloproteinase-1

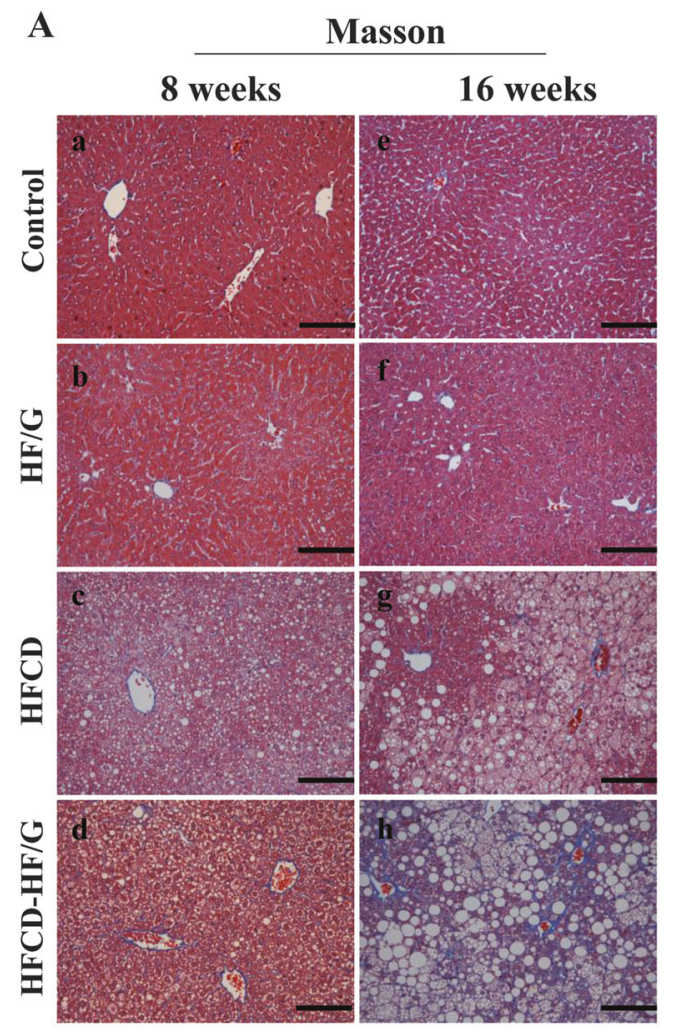

B
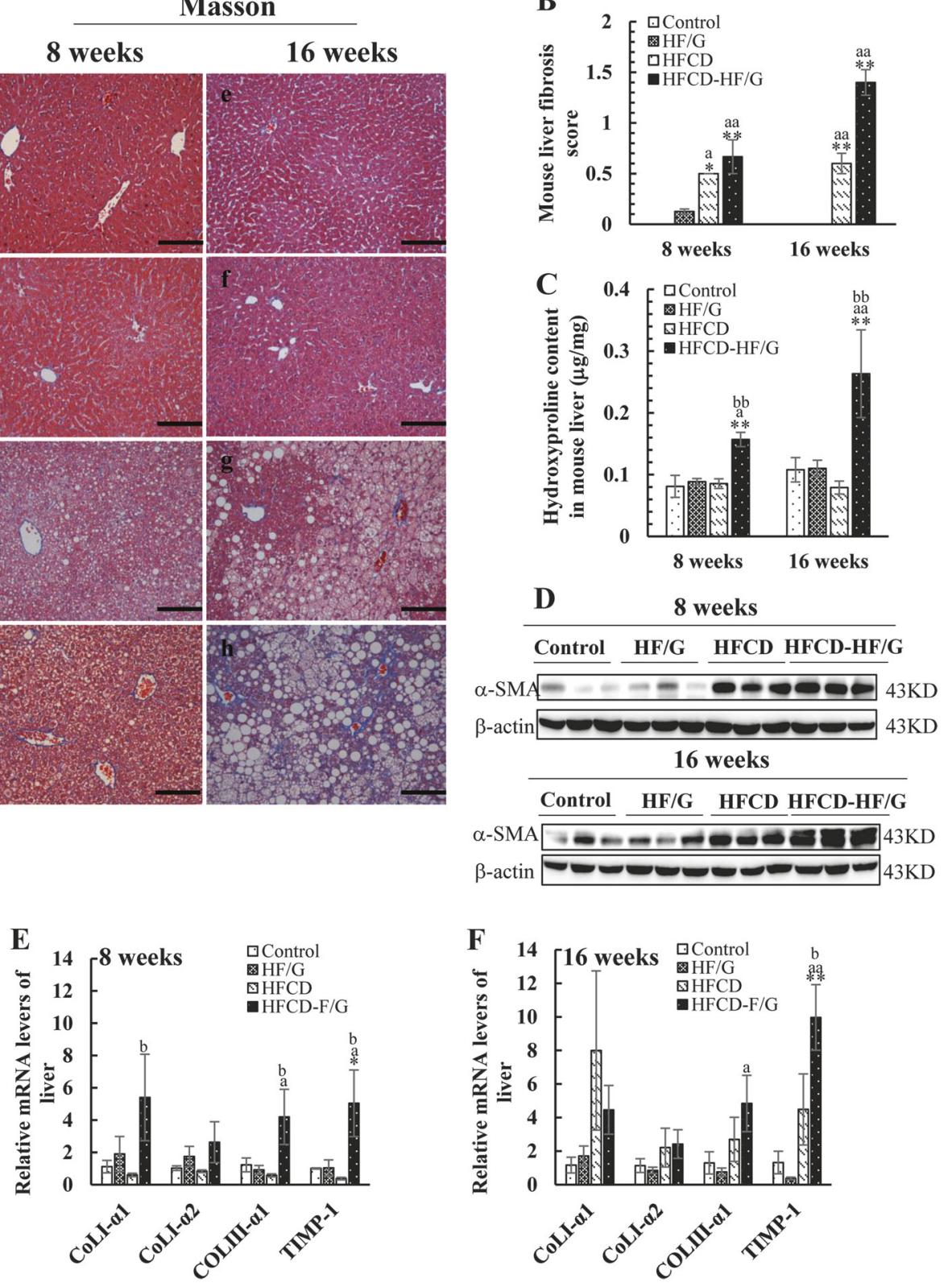

Masson's trichrome staining (Fig. 6B, C, Supplemental Fig. 1D). Smooth muscle $\alpha$-actin ( $\alpha$-SMA) protein levels (Fig. 6D) and liver mRNA levels of procollagen type-I (Col-I $\alpha 1$ and Col-I $\alpha 2$ ), procollagen type-III (Col-III $\alpha 1$ ), and tissue inhibitor of metalloproteinase-1 (TIMP-1) supported the enhanced hepatic fibrosis in the HFCD-HF/G group at 8 and 16 weeks (Fig. 6E, F) compared to the control group. In addition, $\alpha$-SMA protein levels (Fig. 6D) and liver mRNA levers of Col-I $\alpha 1$, Col-III $\alpha 1$, and TIMP-1 in HFCD at 16 weeks (Fig. 6F) were higher than the control, but much less remarkable in comparison to the HFCD$\mathrm{HF} / \mathrm{G}$ group. No significant hepatic fibrosis was observed in histology and biochemical tests from mice fed HF/G. Densitometric analysis of Western blot images of $\alpha$-SMA is shown in Supplemental Fig. 1C and verifies the findings. In summary, it is clear that no fibrosis was developed in mice fed HF/G lone; marked hepatic fibrosis was demonstrated in mice fed HFCD-HF/G diet at both 8 and 16 weeks with progressively enhanced extent. Mild fibrosis was seen in histology with increased semi-quantitative score in the mice fed HFCD diet at 16 weeks, but with no significant increase in hydroxyproline content.

\section{Critical enzymes and nuclear receptors involved in the lipid metabolism}

In order to investigate whether nuclear receptors which are involved in the regulation of lipid metabolism and 
Fig. 7 Expression levels of critical enzymes and transcription factors involved in fatty acid synthesis and $\beta$ oxidation. A, B Total protein (A) and nuclear protein $(\mathbf{B})$ levels of SREBP-1c, ChREBP, FXR, LXR- $\alpha$, PPAR- $\alpha$, PPAR- $\gamma$, and Fasn (total protein) at 8 and 16 weeks were analyzed by western blot analysis $(n=3)$. C, D mRNA levels of Fasn, ACC$\beta$, SCD-1, CPT-1, and ACOX-1 at $8(\mathbf{C})$ and $16(\mathbf{D})$ weeks were determined by quantitative RTPCR analysis $(n=3) . * p<0.05$ and $* * p<0.01$ compared to the controls. SREBP-1c sterol regulatory element binding protein-1c, ChREBP carbohydrate response element binding protein, $F X R$ farnesoid $\mathrm{X}$ receptor, $L X R-\alpha$ liver $\mathrm{X}$ receptor- $\alpha, P P A R-\alpha$ peroxisome proliferator-activated receptor- $\alpha$, $P P A R-\gamma$ peroxisome

proliferators-activated receptor$\gamma$, Fasn fatty acid synthase, $T B P$ TATA-binding protein as a nuclear protein, $A C C-\beta$ acetylCoA carboxylase- $\beta, S C D-1$ stearoyl-CoA desaturase-1, CPT-1 carnitine palmitoyltransferase-1, ACOX-1 acyl-CoA oxidase-1
A

\begin{tabular}{ll}
\multicolumn{2}{c}{8 weeks } \\
\hline Control & HF/G HFCD HFCD-HF/G \\
\end{tabular}

SREBP-1c - - -

ChREBP --

FXR

LXR- $\alpha$ $---\infty-\infty-\infty-\infty-\infty$

PPAR- $\alpha$ $--\cdots-0000-00$

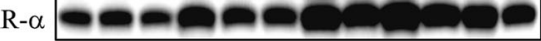

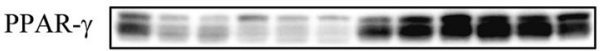

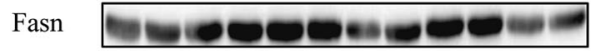

$\beta$-actin

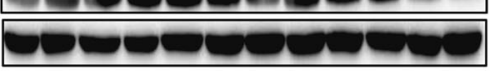

B

8 weeks
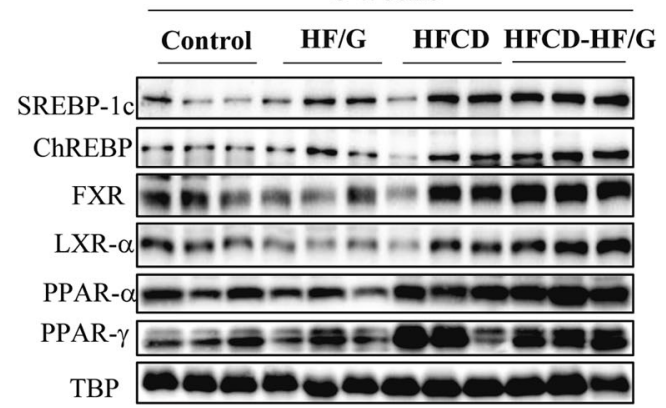

C

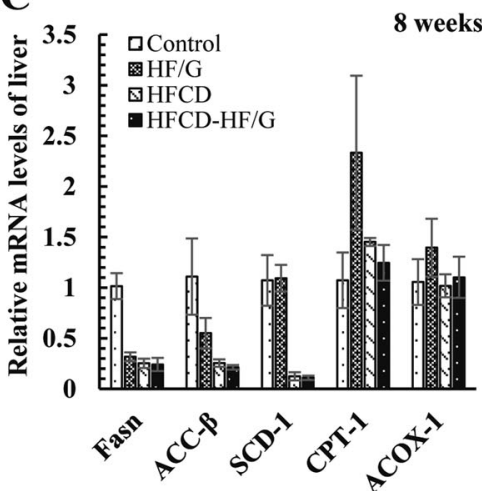

16 weeks

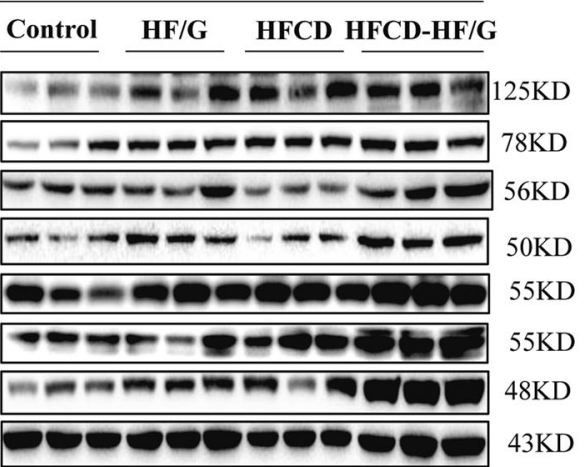

16 weeks

Control HF/G HFCD HFCD-HF/G

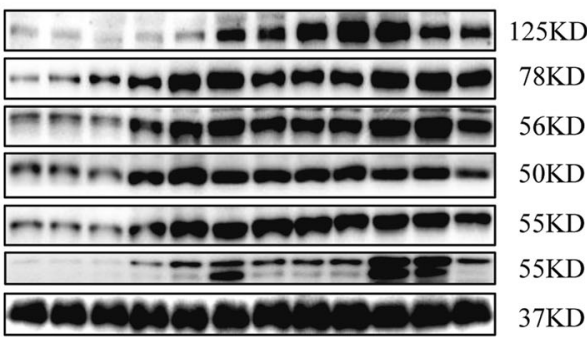

D

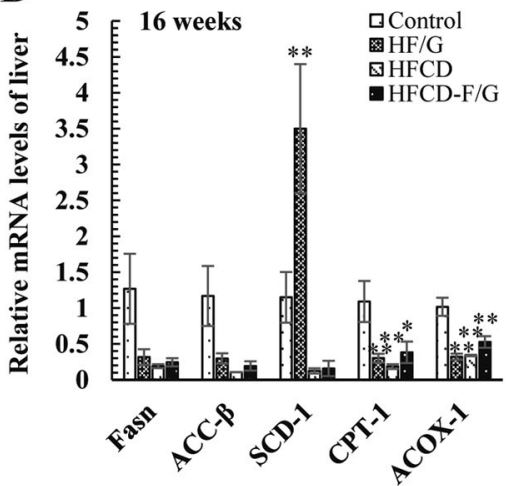

homeostasis, total protein levels of sterol regulatory element binding protein-1c (SREBP-1c), carbohydrate response element binding protein (ChREBP), farnesoid X Receptor (FXR), liver $\mathrm{X}$ receptor- $\alpha$ (LXR- $\alpha)$, peroxisome proliferator-activated receptor- $\alpha$ (PPAR- $\alpha$ ), and peroxisome proliferators-activated receptor- $\gamma$ (PPAR- $\gamma$ ) were determined by Western blot analysis. As shown in Fig. 7A, SREBP-1c, ChREBP, FXR, LXR- $\alpha$, PPAR- $\alpha$, and PPAR- $\gamma$ were found to increase significantly at 8 and 16 weeks in the HFCD-HF/G group, and protein levels of fatty acid synthase (Fasn) were elevated only at 16 weeks. Nuclear protein levels of SREBP-1c, ChREBP, FXR, LXR- $\alpha$, PPAR- $\alpha$, and PPAR- $\gamma$ in the HFCD-HF/G group were obviously elevated compared to the controls at both time points (Fig. 7B). In addition, HFCD feeding caused increased total protein levels of LXR- $\alpha$, PPAR- $\alpha$, and PPAR- $\gamma$ at 8 weeks; SREBP-1c, ChREBP, PPAR- $\alpha$, PPAR- $\gamma$ and Fasn at 16 weeks (Fig. 7A). HFCD feeding augmented nuclear SREBP-1c, PPAR- $\alpha$, and PPAR- $\gamma$ levels at 8 weeks; SREBP-1c, ChREBP, FXR, LXR- $\alpha$, PPAR- $\alpha$, and PPAR- $\gamma$ levels at 16 weeks (Fig. 7B). HF/G feeding elicited an increase in total protein levels of SREBP-1c, FXR, LXR- $\alpha$, PPAR- $\alpha$, and Fasn at 8 weeks; SREBP-1c, ChREBP, LXR$\alpha$, PPAR- $\alpha$, and Fasn at 16 weeks (Fig. 7A); nuclear protein levels of SREBP-1c at 8 weeks; SREBP-1c, ChREBP, FXR, LXR- $\alpha$, PPAR- $\alpha$, and PPAR- $\gamma$ at 16 weeks (Fig. 7B) although these mice had nearly normal glucose tolerant test, fasting serum insulin levels, and HOMA-IR index, as well as minimal steatotic changes in liver histology. Densitometric analysis of Western blot images is shown in 
Fig. 8 Inflammasome activation in mice under different diet feeding. A, B Representative confocal micrographs of ALB, $\alpha$-SMA, and CD163 with NLRP-1 (A) and NLRP-3 coimmunofluorescent staining (B) at 16 weeks. For a-d, ALB was stained in red and NLRP1/3 was stained in green; for $\mathrm{e}-\mathrm{h}, \alpha-$ SMA was stained in green and NLRP1/3 was stained in red; for $\mathrm{i}-1, \mathrm{CD} 163$ was stained in green and NLRP1/3 was in red. Images were taken at original magnification $(\times 400)$. Scale bars $=50 \mu \mathrm{m}$. C Western blot analysis of NLRP-1, NLRP-3, cleaved IL-1 $\beta$, and cleaved caspase- 1 protein levels at 8 and 16 weeks $(n=3)$. NLRP1 NODlike receptor-1, NLRP3 NODlike receptor-3, $A L B$ albumin, $\alpha$-SMA smooth muscle $\alpha$-actin, CD163 cluster of differentiation 163
A
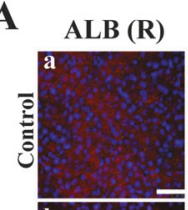

a-SMA (G)
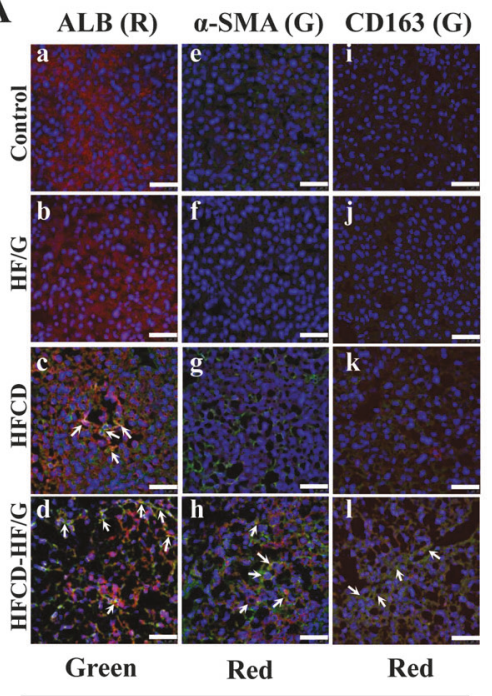

NLRP-1

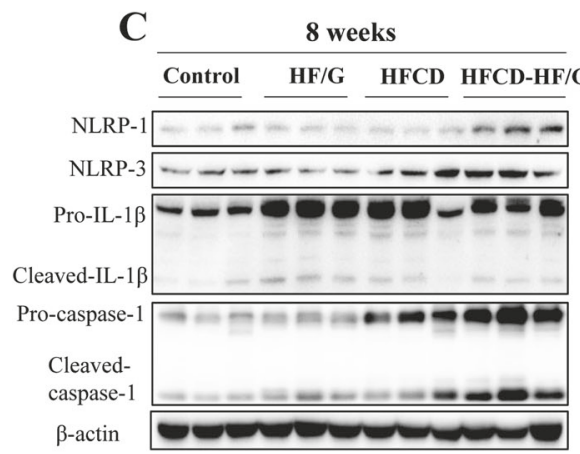

B

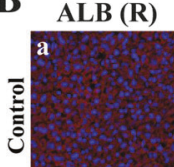

a-SMA (G)

CD163
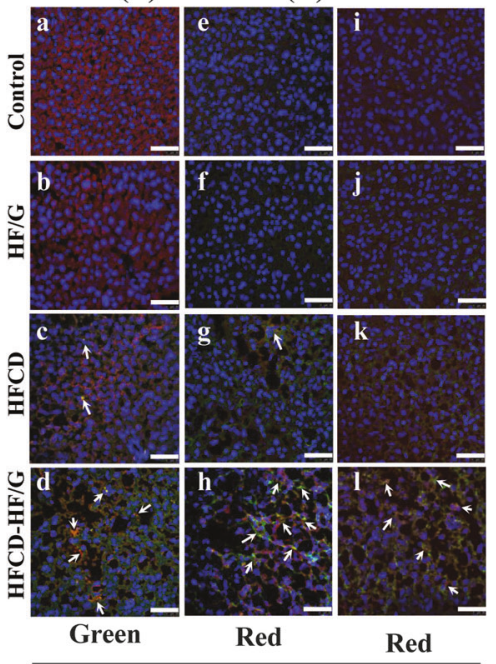

NLRP-3
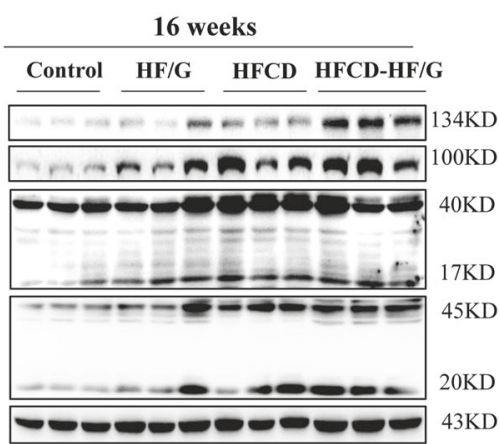

Supplemental Fig. 2, and verifies the findings. In summary, HFCD-HF/G feeding up-regulated SREBP-1c, ChREBP, FXR, LXR- $\alpha$, PPAR- $\alpha$, and PPAR- $\gamma$ significantly at 8 and 16 weeks in fractions of total and nuclear protein. In addition, high-fat diet increased LXR- $\alpha$, PPAR- $\alpha$, and PPAR- $\gamma$ levels at 8 weeks, and SREBP-1c, ChREBP, FXR, and Fasn protein levels were elevated obviously only at a late stage, whereas HF/G feeding only resulted in elevated expression of SREBP-1c, ChREBP, LXR- $\alpha$, PPAR- $\alpha$, and Fasn to some extent.

As shown in Fig. 7C, D, mRNA levels of Fasn and acetyl-CoA carboxylase- $\beta$ (ACC- $\beta$ ) in the HF/G, HFCD, and HFCD-HF/G groups were reduced distinctly at 8 and 16 weeks compared to the controls. However, the Fasn protein levels were increased in HFCD-HF/G fed mice (16 weeks) (Fig. 7A and Supplemental Fig. 2B). In speculation, the discrepancy may be as a result of to negative feedback of mRNA transcription due to the fact that there is already large TG or FFA accumulation in hepatocytes. mRNA levels of stearoyl-CoA desaturase-1 (SCD-1) in the HFCD and HFCD-HF/G groups were decreased obviously to $12 \%$ and $10 \%$ of the controls at 8 weeks and to $11 \%$ and $14 \%$ of the controls at 16 weeks, whereas SCD-1 mRNA levels were increased 3 -fold at 16 weeks in the HF/G group compared to the controls. Carnitine palmitoyltransferase-1 (CPT-1) $(30 \%, 18 \%$, and 38\%) and acyl-CoA oxidase-1 (ACOX-1) (32\%, 34\%, and 53\%) mRNA levels in all three groups declined notably at 16 weeks compared to the controls. In summary, gene levels of Fasn, ACC- $\beta$, SCD-1, CPT-1, and ACOX-1 (16 weeks) were down-regulated by HFCD or HFCD-HF/G feeding, indicating a slow-down fatty acid synthesis and $\beta$-oxydation due to excess fat accumulation in the liver. $\mathrm{HF} / \mathrm{G}$ feeding also elevated expression levels of CPT-1 at 8 weeks; however, expression of these genes was somehow suppressed in a late stage. In addition, SCD-1 gene level was extremely elevated at 16 weeks by $\mathrm{HF} / \mathrm{G}$ feeding in comparison to the controls and other groups, indicating there was an accelerated fatty acid synthesis in mice with HF/G feeding (Fig. 7D).

\section{Inflammasome activation in mice under different diet feeding}

Double immunofluorescent staining indicated that NODlike receptor-1 (NLRP-1) and NLRP-3 inflammasomes were activated in hepatocytes (albumin), hepatic stellate 
cells ( $\alpha$-SMA), and Kupffer cells (CD163) by HFCD-HF/G feeding for 8 weeks (Supplemental Fig. 3A, B) and 16 weeks (Fig. 8A, B). As shown by Western blot analysis in Fig. 8C, increased protein levels of NLRP-1, NLRP-3, cleaved caspase-1, and cleaved interleukin-1 $\beta$ (IL-1 $\beta$ ) documented that the inflammasome pathway was activated in mice fed HFCD-HF/G. It appeared that these two inflammasomes were also activated in hepatocytes by HFCD feeding at 16 weeks (Fig. 8A, B), and that protein levels of NLRP-1, NLRP-3, cleaved caspase-1, and cleaved IL-1 $\beta$ were elevated significantly in the HFCD group compared to the control (Fig. 8C). Densitometric analysis of Western blot images is given in Supplemental Fig. 4 and verifies the findings. In summary, NLRP-1 and NLRP-3 inflammasomes were activated in hepatocytes (Supplemental Fig. 5A, B), hepatic stellate cells (Supplemental Fig. 6A, B), and Kupffer cells (Supplemental Fig. 7A, B) in mice fed HFCD-HF/G at both 8 and 16 weeks. This supports more profound inflammatory response and fibrotic deposition in this group than other groups, and indicates there is a direct effect of inflammasomes on activation of HSCs, which may contribute to more fibrotic degree in this group. Moreover, a late increase in downstream molecules of the inflammasome activation pathway, such as IL- $1 \beta$ and caspase-1, was seen in the HFCD group. However, only elevated IL-1 $\beta$ protein level was seen during HF/G feeding.

\section{Discussion}

Pandemic occurrence of NAFLD worldwide is a serious threat to health of general population and a big economic burden in healthcare to the society. As a progressive stage in the natural history of NAFLD, NASH needs medical intervention, and requires a reliable animal model to assess the efficacy of pharmacotherapeutic intervention, although lifestyle change, dietary restriction, and physical exercise are the cornerstones for initial intervention [6]. Several rodent models, especially available murine models of $\mathrm{NASH}$, are widely used for preclinical investigation of pharmacotherapeutic candidates. However, nutritional composition (fat or cholesterol content, transfat percentage, carbohydrate proportion, and so on), feeding duration and with or without supplementation of fructose and/or glucose vary dramatically in various studies, which results in huge variances in the features of NASH, particularly in extent of steatosis, necroptosis, disturbance of adipokine profile, and inflammatory responses, as well as insulin resistance and hepatic fibrosis progression [1-3]. These are the major endpoints for clinical trials of pharmacological candidates, such as vitamin E, pioglitazone (PGZ), obeticholic acid [20, 21], and those in on-going phase II and III trials, such as an apoptosis signal-regulating kinase-1 (ASK-1) inhibitor
(Selonsertib), a dual antagonist of CCR-2 and -5 (Cenicriviroc) $[22,23]$ and a dual PPAR- $\alpha,-\gamma$ agonist, Elafibranor (GFT505) [24]. It is a big challenge to estimate the efficacy of pharmacotherapeutic candidates in a preclinical setting when no standardized murine NASH model is available. To address this unmet need, we fed mice with a standard high-fat/calorie diet (HFCD), high fructose/glucose $(\mathrm{HF} / \mathrm{G})$ in drinking water separately, or in combination of these two (HFCD-HF/G), which mimics Western fast food and soft drinks with high-sugar content $[12,13]$. The major end-points of NASH were compared, including extent of steatosis, necroptosis, insulin resistance, and fibrotic progression, as well as disturbance in lipid metabolic panels and cytokine, adipokine profile, and inflammatory responses. In addition, insulin receptor signaling defects, nuclear factor or receptors regulating lipogenesis and critical enzymes participating in fatty acid synthesis or $\beta$-oxydation were also evaluated for better understanding of disturbances in nutritional hemostasis and regulation under severe obesity with NASH.

With objective comparison in quantitative variables, such as liver TG, serum ALT, hydroxyproline content, fasting insulin levels, and other parameters, it is clear that $\mathrm{HF} / \mathrm{G}$ feeding increased liver cholesterol, and no significant elevation in liver TG content, obvious necroptosis, inflammatory responses, such as elevation of inflammatory cytokines or adipokines, infiltration of inflammatory cells were observed in the liver. In addition, there is no significant IGTT or fast serum insulin abnormalities, and deposition of fibril collagens. However, HF/G did suppress gene expression levels of INSR, CPT-1, and ACOX-1, without activating inflammasomes. All nuclear receptors/transcription factors (except PPAR- $\alpha$ ) and other critical enzymes (except SCD-1) involved in the lipogenesis and $\beta$-oxydation were unnoticeable. In consistent with its effect in eliciting inflammatory response [25], it appeared that $\mathrm{HF} / \mathrm{G}$ did induce pro-IL- $\beta$ and cleaved IL- $\beta$ at protein levels at both 8 and 16 weeks, but did not cause inflammatory cell infiltration, and did not affect MCP-1 and CCR-2 mRNA levels and NLRP-1, NLRP-3, cleaved caspase-1 protein levels. Therefore, the extent of inflammatory response was not dramatic if there was any. Thus, it is evident from this study that high fructose and glucose in drinking water only caused minimal fat accumulation and metabolic disturbance in regular mice during 16 weeks of feeding, shares less similarity to manifestations seen in NASH patients, and may be non-optimal as a model to study the pathogenesis and efficacy assessment in the treatment of NASH.

In contrast, mice fed high-fat/calorie diet with high fructose/glucose in drinking water (HFCD-HF/G) developed marked steatosis with a nearly seven-fold increase in liver TG content with pronounced necroptosis (5-9-fold in ALT), inflammatory filtration, insulin resistance (1-3-fold 
increase in fast insulin content), progressive fibrosis (2-3fold increase in hydroxyproline content) at 8 weeks and become worsening at 16 weeks. Expression of inflammatory cytokines, such as TNF- $\alpha$, was elevated whereas adipokines, such as leptin and adiponectin, were suppressed in the liver compared to the controls as seen in our previous studies [9, 13]. Moreover, the metabolic profiles indicate a great similarity to manifestations seen in NASH patients, such as high hyperlipidemia (TC, TG, LDL, HDL), hyperglycemia, hyperinsulinemia, insulin resistance with defective insulin receptor signaling, marked subcutaneous and visceral adipose accumulation, body weight gain, and so on. The nuclear receptors or transcription factors, such as SREBP-1c, ChREBP, FXR, PPAR- $\alpha$, PPAR- $\gamma$ [26-29], as well as Fasn protein levels were significantly elevated in the liver of mice fed HFCD-HF/G for 8 weeks and even more profound at 16 weeks. The up-regulation of PPAR- $\alpha$, PPAR- $\gamma$, and FXR in murine models of NASH may be contradictory to the use of agonists of PPAR- $\alpha$, PPAR- $\gamma$, and FXR agonists, such as pioglitazone (PGZ) [21], Elafibranor [24], and obeticholic acid [20] as pharmacotherapeutics in NASH patients, and reflects dissimilarity between murine models and the human disease. However, this phenomenon is consistent with reports from other mouse studies, in which PPAR- $\alpha$ was often found to be down-regulated [30] in MCD diet-fed mice or up-regulated in mice with long-term HFCD-HF/G probably due to its protective mechanism [31]. PPAR- $\gamma$ is often up-regulated in high-fat diet feeding mice, especially when fructose was added [32], and both PPAR- $\gamma$ agonists and antagonists are therapeutic candidates [33] for NASH treatment with a tendency to use pan-agonists for PPAR- $\alpha / \gamma / \delta$ [34]. As mentioned previously, an FXR agonist, obeticholic acid, has been proven to be effective in improving insulin resistance, NAS scores, and fibrosis in phase II clinical trials [20, 35].

In the present study, it is notable that feeding mice HFCD-HF/G for 16 weeks would be as an optimal NASH model in terms of profound steatosis and necroptosis, insulin resistance and progressive fibrosis with remarkable profiles of cytokine and adipokine disturbance, disordered lipid and carbohydrate metabolism and regulation. This model possessed significant inflammatory responses, as evidenced by infiltration of inflammatory cells in the portal triads, increased MCP-1 and CCR-2 mRNA levels, elevated NLRP-1, NLRP-3, IL-1 $\beta$, cleaved caspase-1 protein levels [36], as well as up-regulated TNF- $\alpha$ and IL- $\beta$ mRNA levels in the liver. Suppressed Fasn, ACC- $\beta$, and SCD-1 mRNA levels in these mice indicated a negative feedback regulation in fatty acid synthesis due to excessive fat accumulation in the liver. In consistent, the enzymes involved in fatty acid $\beta$-oxydation, such as CPT- 1 and ACOX-1 gene expression, were remarkably suppressed in this model, indicating the defect in FFA consumption and energy production in damaged hepatocytes [9]. This model was established on regular mice without genetic manipulation and mortality in a duration of nearly 4 months, and is acceptable as a standard for pharmaceutical intervention and pathophysiologic investigation.

In comparison with HFCD-HF/G, HFCD feeding led to marked steatosis, necroptosis, and insulin resistance with minimal fibrosis at 16 weeks; however, the extent is less profound than HFCD-HF/G. In consistent, metabolic abnormalities, cytokine, adipokine profile, fast insulin levels, activation of nuclear receptors or transcription factors highly support a notion that this is a NASH model with significant steatosis, moderate necroptosis, mild insulin resistance with minimal fibrotic response (activation of smooth muscle $\alpha$-actin and enhanced procollagen production but without significant hydroxyproline content increase). This model should be useful merely for evaluation of pharmaceutical candidates which target to minimize lipotoxicity, fat accumulation, and inflammatory responses, but does not take hepatic fibrosis into serious account. The reason is that hydroxyproline content is proportional to actual collagen content and is the most reliable variable in reflecting total amount of net collagen deposition in the liver at the time of measurement [37].

It was our initial interest to determine whether the combination of high-fat/calorie diet plus high fructose and glucose would have synergic effects in accelerating the development of lipotoxicity, necroptosis, inflammatory response, insulin resistance, and fibrotic progression [3]. In comparison with HFCD, mice fed HFCD-HF/G did exhibit more profound characteristics and earlier appearance in all these aspects as end-points of severity assessment of NASH progression since HF/G alone did not give rise to phenotypic features of NASH during 16 weeks of feeding. It is evident that a significant increase in SCD-1 mRNA levels (Fig. 7D) and protein levels of cleaved IL-1 $\beta$ (Fig. 8C, Supplemental Fig. 4B), as well as nuclear receptors or transcription factors, such as ChREBP, FXR, LXR- $\alpha$, PPAR- $\alpha$ (Fig. 7B, Supplemental Fig. 2D) were seen in mice with 16 weeks of HF/G feeding, indicating elevated lipogenesis and inflammatory response in these mice. However, it is premature to draw such a conclusion due to the fact that most end-point variables or semi-quantitative scores of histopathologic observation could not provide such an accuracy that allows to determine the additive or synergic effects and that biochemical and histopathologic variables did not provide evidence of significant obese, hepatic steatosis, inflammation, necroptosis, fibrosis, and insulin resistance in mice fed HF/G over time.

It is well acceptable that there is significant inflammatory response in NASH in patients and a dual antagonist of CCR-2 and -5 (Cenicriviroc) has been tested in clinical 
trials [22, 23]. In our murine models, mRNA levels of MCP-1 and CCR-2 mRNA levels, NLRP-1, NLRP-3, IL$1 \beta$, cleaved caspase- 1 protein levels, as well as TNF- $\alpha$ and IL-1 $\beta$ mRNA levels were elevated in the liver. In other studies, no significant elevation in TNF- $\alpha$, IL-6, and interferon- $\beta$ (IFN- $\beta$ ) levels in the liver was found in a mouse model of NASH over 8-24 weeks of high-fat diet feeding [38], but was elevated at 8 weeks of high-fat diet feeding in a different study [39]. Serum TNF- $\alpha$, IL-6, and IL-10 levels were rapidly elevated in mice after endotoxin injection [40]. Therefore, there exist discrepancies between mRNA levels and protein levels of these inflammatory factors in different studies, and would be more reliable to have their levels measured at both mRNA and protein levels in the liver and serum at the same time when anti-inflammatory agents are evaluated as therapeutics in the treatment of NASH.

Taking together, the findings of this study demonstrate that feeding HFCD-HF/G resulted in a reliable NASH model in mice representing striking necroptosis, steatosis, fibrosis, and insulin resistance with a highly similar profile of disordered lipid metabolism and adipokines, and HFCD only caused significant NASH features in histopathology and metabolic profiles at a late stage with less severe extent, whereas HF/G feeding barely caused minimal fat accumulation and metabolic disturbance in mice, and did not recapture NASH at histopathology and metabolic profile. The characterization of murine NASH model provides a means of standardization in end-point variables, and offers a cross-board comparison of pharmacologic candidates in effectiveness and adverse effects.

Acknowledgements The authors are grateful to Ms. Xiao Guo in the Joint Live Small Animal Imaging Laboratory of Fudan University Shanghai Medical College-PerkinElmer Company, for her technical support in the use of high-resolution X-ray microCT scanning. The authors thank Ms. Ke Qiao in the Department of Medical Microbiology and Parasitology, Fudan University School of Basic Medical Sciences for her technical assistance in the use of confocal microscope.

Funding This work is supported by the National Natural Science Foundation of China (NSFC \#81272436 \& 81572356 to J.W.) and (NSFC \#81470857 to X.-P.L.), as well as Shanghai Commission of Sciences and Technologies (\#16140903700), and the Ministry of Science \& Technology of China (\#2016YFE0107400) to J.W.

\section{Compliance with ethical standards}

Conflict of interest The authors declare that they have no conflict of interest.

\section{References}

1. Maher JJ. New insights from rodent models of fatty liver disease. Antioxid Redox Signal. 2011;15:535-50.

2. Hebbard L, George J. Animal models of nonalcoholic fatty liver disease. Nat Rev Gastroenterol Hepatol. 2011;8:35-44.
3. Ibrahim SH, Hirsova P, Malhi H, Gores GJ. Animal models of nonalcoholic steatohepatitis: eat, delete, and inflame. Dig Dis Sci. 2016;61:1325-36.

4. Imajo K, Yoneda M, Kessoku T, et al. Rodent models of nonalcoholic fatty liver disease/nonalcoholic steatohepatitis. Int J Mol Sci. 2013;14:21833-57.

5. Rinella ME, Green RM. The methionine-choline deficient dietary model of steatohepatitis does not exhibit insulin resistance. J Hepatol. 2004;40:47-51.

6. Diehl AM, Day C. Cause, pathogenesis, and treatment of nonalcoholic steatohepatitis. N Engl J Med. 2017;377:2063-72.

7. Schattenberg JM, Galle PR. Animal models of non-alcoholic steatohepatitis: of mice and man. Dig Dis. 2010;28:247-54.

8. Spruss A, Kanuri G, Wagnerberger S, et al. Toll-like receptor 4 is involved in the development of fructose-induced hepatic steatosis in mice. Hepatology. 2009;50:1094-104.

9. Adkins Y, Schie IW, Fedor D, et al. A novel mouse model of nonalcoholic steatohepatitis with significant insulin resistance. Lab Invest. 2013;93:1313-22.

10. Denda A, Kitayama W, Kishida H, et al. Development of hepatocellular adenomas and carcinomas associated with fibrosis in C57BL/6J male mice given a choline-deficient, L-amino aciddefined diet. Jpn J Cancer Res. 2002;93:125-32.

11. Dowman JK, Hopkins LJ, Reynolds GM, et al. Development of hepatocellular carcinoma in a murine model of nonalcoholic steatohepatitis induced by use of a high-fat/fructose diet and sedentary lifestyle. Am J Pathol. 2014;184:1550-61.

12. Asgharpour A, Cazanave SC, Pacana T, et al. A diet-induced animal model of non-alcoholic fatty liver disease and hepatocellular cancer. J Hepatol. 2016;65:579-88.

13. Xu G, Ye J, Liu XJ, et al. Activation of pluripotent genes in hepatic progenitor cells in the transition of nonalcoholic steatohepatitis to pre-malignant lesions. Lab Invest. 2017;97: 1201-17.

14. Metzinger MN, Miramontes B, Zhou P, et al. Correlation of $\mathrm{X}$-ray computed tomography with quantitative nuclear magnetic resonance methods for pre-clinical measurement of adipose and lean tissues in living mice. Sensors (Basel). 2014;14: $18526-42$.

15. Zhu J, Wu J, Frizell E, et al. Rapamycin inhibits hepatic stellate cell proliferation in vitro and limits fibrogenesis in an in vivo model of liver fibrosis. Gastroenterology. 1999;117:1198-204.

16. Kleiner DE, Brunt EM, Van Natta M, et al. Design and validation of a histological scoring system for nonalcoholic fatty liver disease. Hepatology. 2005;41:1313-21.

17. Wu J, Liu L, Yen RD, et al. Liposome-mediated extracellular superoxide dismutase gene delivery protects against acute liver injury in mice. Hepatology. 2004;40:195-204.

18. Lingala $\mathrm{S}$, Cui YY, Chen X, et al. Immunohistochemical staining of cancer stem cell markers in hepatocellular carcinoma. Exp Mol Pathol. 2010;89:27-35.

19. Chen X, Lingala S, Khoobyari S, et al. Epithelial mesenchymal transition and hedgehog signaling activation are associated with chemoresistance and invasion of hepatoma subpopulations. J Hepatol. 2011;55:838-45.

20. Neuschwander-Tetri BA, Loomba R, Sanyal AJ, et al. Farnesoid $\mathrm{X}$ nuclear receptor ligand obeticholic acid for non-cirrhotic, nonalcoholic steatohepatitis (FLINT): a multicentre, randomised, placebo-controlled trial. Lancet. 2015;385:956-65.

21. Sanyal AJ, Chalasani N, Kowdley KV, et al. Pioglitazone, vitamin $\mathrm{E}$, or placebo for nonalcoholic steatohepatitis. N Engl J Med. 2010;362:1675-85.

22. Friedman SL, Ratziu V, Harrison SA, et al. A randomized, placebo-controlled trial of cenicriviroc for treatment of nonalcoholic steatohepatitis with fibrosis. Hepatology 2018;67: 1754-67. 
23. Loomba R, Lawitz E, Mantry PS, et al. The ASK1 inhibitor selonsertib in patients with nonalcoholic steatohepatitis: a randomized, phase 2 trial. Hepatology 2017. https://doi.org/10.1002/ hep.29514.

24. Ratziu V, Harrison SA, Francque S, et al. Elafibranor, an agonist of the peroxisome proliferator-activated receptor-alpha and -delta, induces resolution of nonalcoholic steatohepatitis without fibrosis worsening. Gastroenterology. 2016;150:1147-59 e5.

25. Bergheim I, Weber S, Vos M, et al. Antibiotics protect against fructose-induced hepatic lipid accumulation in mice: role of endotoxin. J Hepatol. 2008;48:983-92.

26. Dubuquoy C, Robichon C, Lasnier F, et al. Distinct regulation of adiponutrin/PNPLA3 gene expression by the transcription factors ChREBP and SREBP1c in mouse and human hepatocytes. J Hepatol. 2011;55:145-53.

27. Arab JP, Karpen SJ, Dawson PA, Arrese M, Trauner M. Bile acids and nonalcoholic fatty liver disease: molecular insights and therapeutic perspectives. Hepatology. 2017;65:350-62.

28. Filhoulaud G, Guilmeau S, Dentin R, Girard J, Postic C. Novel insights into ChREBP regulation and function. Trends Endocrinol Metab. 2013;24:257-68

29. Teodoro JS, Rolo AP, Palmeira CM. Hepatic FXR: key regulator of whole-body energy metabolism. Trends Endocrinol Metab. 2011;22:458-66.

30. Loyer X, Paradis V, Henique C, et al. Liver microRNA-21 is overexpressed in non-alcoholic steatohepatitis and contributes to the disease in experimental models by inhibiting PPARalpha expression. Gut. 2016;65:1882-94.

31. Montagner A, Polizzi A, Fouche E, et al. Liver PPARalpha is crucial for whole-body fatty acid homeostasis and is protective against NAFLD. Gut. 2016;65:1202-14.
32. Schultz A, Neil D, Aguila MB, Mandarim-de-Lacerda CA. Hepatic adverse effects of fructose consumption independent of overweight/obesity. Int J Mol Sci. 2013;14:21873-86.

33. Zhang Y, Cui Y, Wang XL, et al. PPARalpha/gamma agonists and antagonists differently affect hepatic lipid metabolism, oxidative stress and inflammatory cytokine production in steatohepatitic rats. Cytokine. 2015;75:127-35.

34. Wettstein G, Luccarini JM, Poekes L, et al. The new-generation pan-peroxisome proliferator-activated receptor agonist IVA337 protects the liver from metabolic disorders and fibrosis. Hepatol Commun. 2017;1:524-37.

35. Mudaliar S, Henry RR, Sanyal AJ, et al. Efficacy and safety of the farnesoid $X$ receptor agonist obeticholic acid in patients with type 2 diabetes and nonalcoholic fatty liver disease. Gastroenterology. 2013;145:574-82 e1.

36. Szabo G, Csak T. Inflammasomes in liver diseases. J Hepatol. 2012;57:642-54.

37. Woessner JF Jr. The determination of hydroxyproline in tissue and protein samples containing small proportions of this imino acid. Arch Biochem Biophys. 1961;93:440-7.

38. Zhang RN, Pan Q, Zhang Z, et al. Saturated fatty acid inhibits viral replication in chronic hepatitis B virus infection with nonalcoholic fatty liver disease by toll-like receptor 4-mediated innate immune response. Hepat Mon. 2015;15:e27909.

39. Zheng H, Li S, Ma L, et al. A novel agonist of PPAR-gamma based on barbituric acid alleviates the development of non-alcoholic fatty liver disease by regulating adipocytokine expression and preventing insulin resistance. Eur J Pharmacol. 2011;659:244-51.

40. Komine S, Akiyama K, Warabi E, et al. Exercise training enhances in vivo clearance of endotoxin and attenuates inflammatory responses by potentiating Kupffer cell phagocytosis. Sci Rep. 2017;7:11977. 